\title{
AN EXPERIMENT MANAGEMENT COMPONENT FOR THE WBCSIM PROBLEM SOLVING ENVIRONMENT
}

\author{
by \\ Jiang Shu \\ Thesis submitted to the Faculty of the \\ Virginia Polytechnic Institute and State University \\ in partial fulfillment of the requirements for the degree of
}

MASTER OF SCIENCE

in

Computer Science

APPROVED:

Layne T. Watson

Chair of Advisory Committee

Frederick A. Kamke

Naren Ramakrishnan

October 1, 2002

Blacksburg, Virginia

Key words: Experiment management, problem solving environment, computing environment, wood-based composite materials, database management, visualization, optimization.

Copyright 2002, Jiang Shu 


\title{
AN EXPERIMENT MANAGEMENT COMPONENT FOR THE WBCSIM PROBLEM SOLVING ENVIRONMENT
}

\author{
by \\ Jiang Shu \\ (ABSTRACT)
}

This thesis describes a computing environment WBCSim and its experiment management component. WBCSim is a web-based simulation system used to increase the productivity of wood scientists conducting research on wood-based composite and material manufacturing processes. This experiment management component integrates a web-based graphical front end, server scripts, and a database management system to allow scientists to easily save, retrieve, and perform customized operations on experimental data. A detailed description of the system architecture and the experiment management component is presented, along with a typical scenario of usage. 


\section{ACKNOWLEDGEMENTS}

I would like to express my appreciation for the support and guidance that I received from my advisor Dr. Layne T. Watson in the past two years. Also, I want to thank the other two members of my advisory committee, Dr. Frederick A. Kamke and Dr. Naren Ramakrishnan, for their sincere support and advice.

Furthermore, I would like to thank Balazs G. Zombori for his work on mat formation modeling, hot compression modeling, and composite material analysis modeling (documented

in Chapter 2). Moreover, I thank Alex Verstak for his work on the telnet server (documented in Chapter 5) in WBCSim. I would also thank Dr. Clifford A. Shaffer and Dr. Calvin J. Ribbens for their effort in organizing research meetings to help students collaborate with each other.

This work was supported in part by a Virginia Polytechnic Institute and State University 1997 ASPIRES grant, USDA Grant 97-35504-4697, and DOE contract DE-AC04-95AL9727391830. 


\section{TABLE OF CONTENTS}

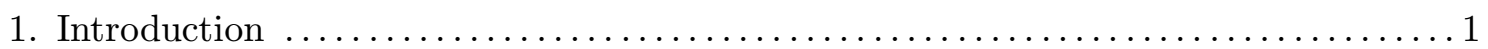

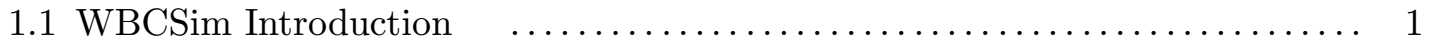

1.2 Experiment Management Component Introduction $\quad \ldots \ldots \ldots \ldots \ldots \ldots \ldots . \ldots 2$

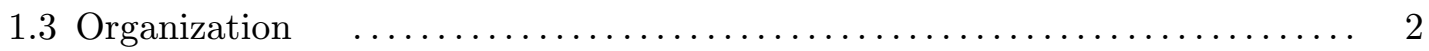

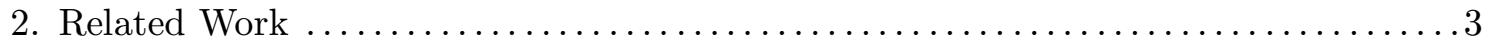

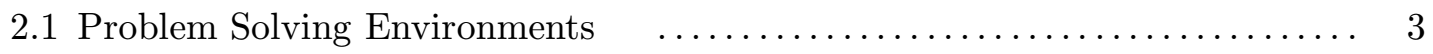

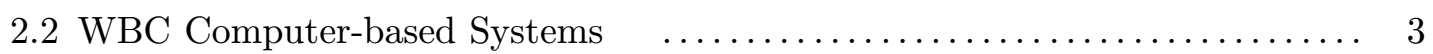

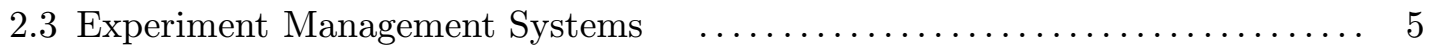

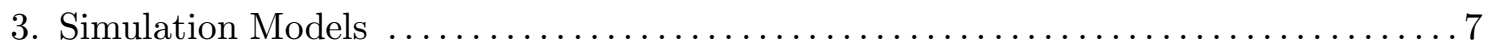

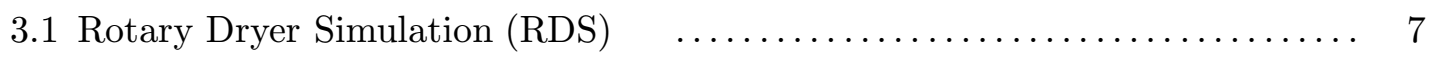

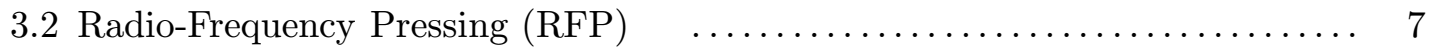

3.3 Oriented Strandboard Mat Formation (OSB) $\ldots \ldots \ldots \ldots \ldots \ldots \ldots \ldots \ldots$

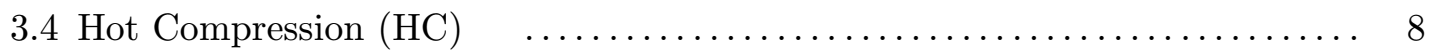

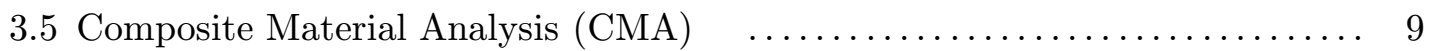

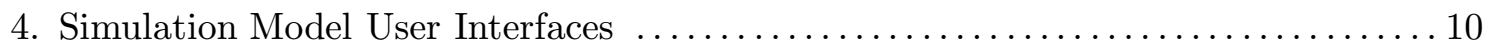

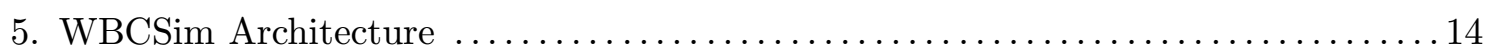

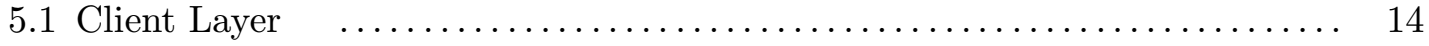

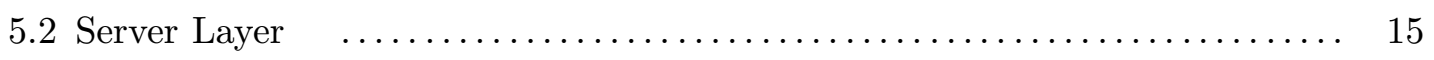

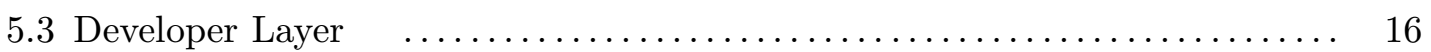

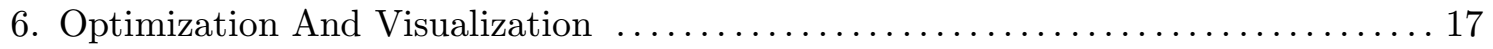

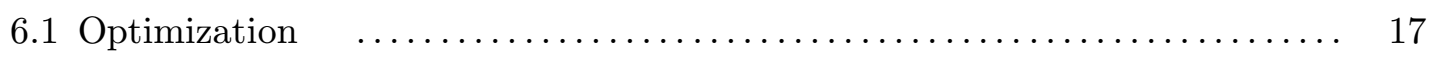

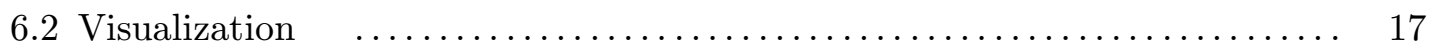

7. Rationale For Experiment Management $\ldots \ldots \ldots \ldots \ldots \ldots \ldots \ldots \ldots \ldots \ldots \ldots \ldots \ldots \ldots \ldots \ldots \ldots$

8. Experiment Management Component Architecture ......................... 22

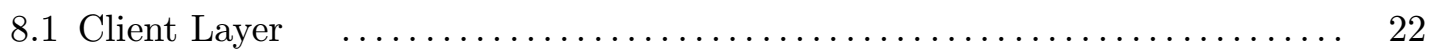

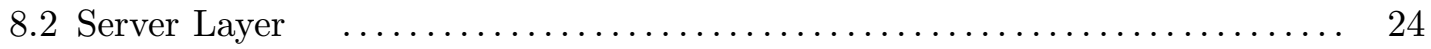

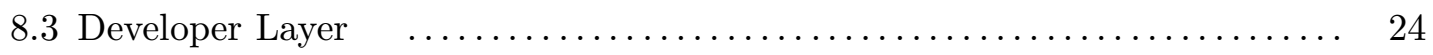

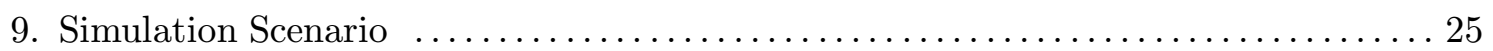

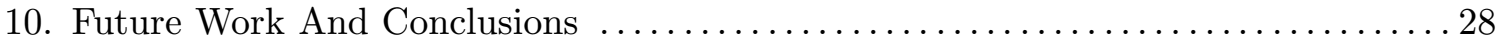

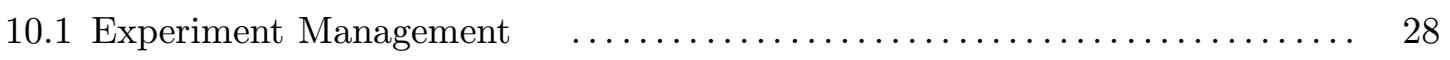

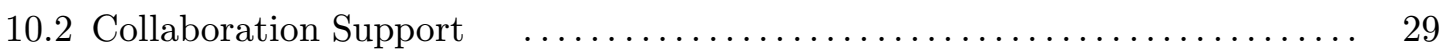

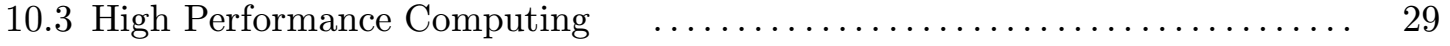




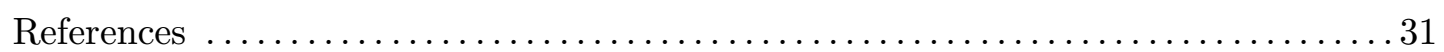

Appendix A: Perl Subroutine gencontourplot ........................... 34

Appendix B: Perl Code Example of WhirlGif from gen3Dplot . .............. 36

Appendix C: Perl OSB Save Wrapper osbsave.pl ....................... 38

Appendix D: Perl OSB Load Wrapper osbload.pl .................... 47

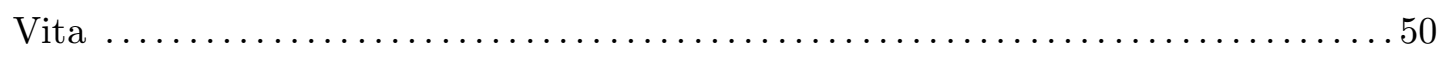

\section{LIST OF FIGURES}

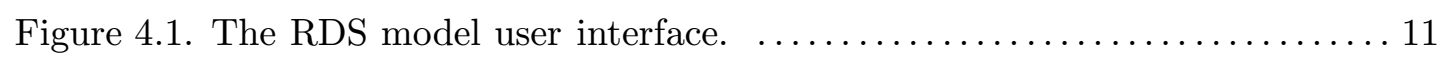

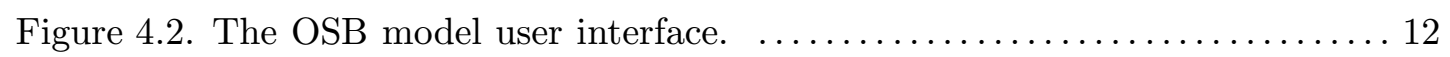

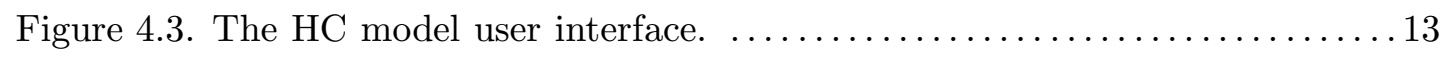

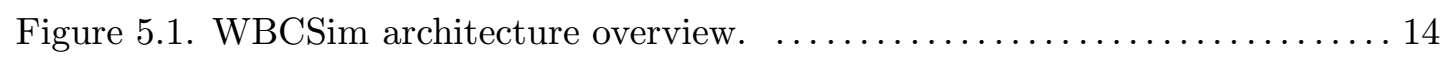

Figure 6.1. VRML pressure graph showing the variation of pressure with increasing distance from the laminate surface and with time (the receding axis) $\ldots \ldots \ldots \ldots 18$ Figure 6.2. Visualization of the three-layer random flake mat: the area of the mat is $450 \times 450 \mathrm{~mm}$. Only a fraction of the flakes are shown, and the shading designates the

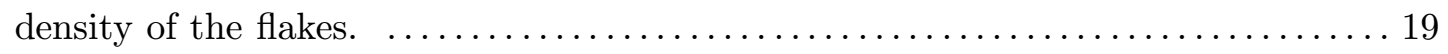

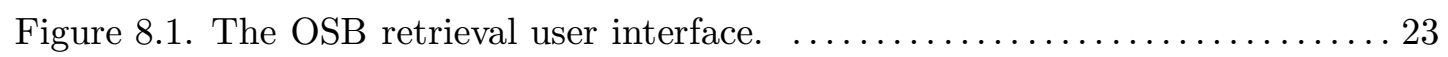

Figure 8.2. The OSB Void Fractions/Contact Area comparison detail user interface. 23

Figure 9.1. The OSB Void Fraction/Contact Area comparison result from two simulation runs. It shows "space/mat" (SM) volume and "contact area" (CA) from those two

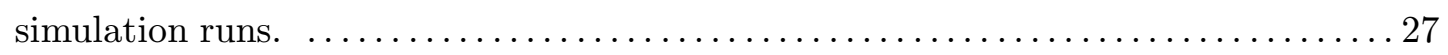




\section{Chapter 1: INTRODUCTION}

Over the past few years, increased attention has been given to the way scientists generate, store, and manage experimental data. Usually, scientists are easily able to generate and store several megabytes of data per experiment. However, they often lack adequate experiment management tools that are not only powerful enough to capture the complexity of the experiments but, at the same time, are natural and intuitive to the non-expert [18]. Therefore, scientists often rely on tagged folders and directory hierarchies to separate and organize experiment data; some scientists have even gone back to the use of paper notebooks to track their data. This situation obviously hinders the productivity of many experimental groups.

In order to address this problem, an experiment management component has been developed for the WBCSim problem solving environment (PSE). This experiment management (EM) component integrates a web-based graphical front end, server scripts, and a database management system to allow scientists to easily save, retrieve, and perform customized operations on experimental data.

\subsection{WBCSim Introduction}

WBCSim is a problem solving environment that increases the productivity of wood scientists conducting research on wood-based composite (WBC) materials and manufacturing processes. It integrates Fortran 90 simulation codes with a Web-based graphical front end, an optimization tool, and various visualization tools. The WBCSim project was begun in 1997 with support from USDA, Department of Energy, and Virginia Polytechnic Institute and State University (VPI). It has since been used by students in several wood science classes, by graduate students and faculty, and by researchers at several forest products companies. User feedback has resulted in numerous changes to the interface and underlying models, and was the major impetus for adding experiment management. Replacing the batch file mode of use by the Web interface, and supporting optimization for manufacturing process design, have had a major impact on the productivity of wood scientists using the analysis codes in WBCSim.

Goel et al. [14], [13] describe an early version of WBCSim. Since then, WBCSim has evolved, taking different approaches to its architecture, adding more sophisticated models, and switching from an experiment-oriented to manufacturer-oriented approach. However, the application's original goals remain the same: (1) to increase the productivity of WBC research and manufacturing groups by improving their software environment, and (2) to continue serving as an example for the design, construction, and evaluation of small-scale PSEs.

Goel et al. [14] give a more complete description of what constitutes a PSE and why WBCSim is a PSE. In general, a PSE provides an integrated set of high-level facilities 
that support users engaged in solving problems from a proscribed domain [11]. Also, a PSE commonly addresses the following issues: Internet accessibility to legacy codes, visualization, experiment management, multidisciplinary support, collaboration support, optimization, high performance computing, usage documentation, preservation of expert knowledge, recommender systems, and integration [27]. WBCSim qualifies as a PSE for the following reasons: it makes legacy simulation codes available via the World Wide Web; it is equipped with visualization and optimization tools; it is multidisciplinary; it has an experiment management component; furthermore it will soon be augmented with collaboration support and high performance computing.

\subsection{Experiment Management Component Introduction}

Despite its multifunctional ability of running simulation experiments and generating and storing data, WBCSim needs an adequate tool to manage its execution and experiment data, and to provide additional features such as experiment searching, comparison, and other data mining techniques. Therefore, such an experiment management component was developed for WBCSim. In short, the intent was to implement a database management system (DBMS) to store simulation inputs and outputs at the server end, provide helpful graphical interfaces to facilitate user access at the client side, and then use some scripts to connect all of these various components.

\subsection{Organization}

Chapters are organized as follows. Chapter 2 reviews some related work in PSEs, WBC computer-based systems, and some experiment management systems. Chapter 3 describes the five simulation models currently supported by WBCSim. Chapter 4 elaborates on the WBCSim user interface, the model interface similarities, and also the unique aspects of each. Chapter 5 explains the various architecture layers of WBCSim, and also the new WBCSim telnet communication connection scheme. Chapter 6 explains the WBCSim optimization tool and the visualization tools involved. Chapter 7 gives the motivation for developing the EM component. Chapter 8 elaborates on the EM component and how the EM component fits in the WBCSim architecture. Chapter 9 demonstrates a typical scenario using this EM component. Chapter 10 outlines some future directions for this EM component and WBCSim, and also draws conclusions. 


\section{Chapter 2: RELATED WORK}

There are a dozen or so problem-specific PSEs developed for various application domains, and several computer-based mathematical models were developed to solve particular problems in the wood-based composites industry. However, no work known to the authors addresses the integration of WBC mathematical models with a problem solving environment. WBCSim is meant to fill this gap and provide a valuable tool for the wood-based composites industry.

\subsection{Problem Solving Environments}

Goel et al. [14] describe some early PSEs such as ELLPACK and its descendents [3] for solving two and three dimensional elliptic partial differential equations (PDE), SCIRun [25] developed to interactively compose, execute, and control a large-scale computer simulation, and Linear System Analyzer [7] for manipulating and solving large-scale sparse linear systems of equations. Since then, several new PSEs have been introduced. Gismo [8], created at Washington University, is an object-oriented Monte Carlo package for modeling all aspects of a satellite's design and performance. It has played a significant role in the design of the Gamma Ray Large Area Space Telescope, the successor to the Compton Gamma Ray Observatory that was launched into space in 1991 to explore the gamma ray portion of the electromagnetic spectrum in astrophysics. VizCraft [12], developed at VPI, provides a graphical user interface for a widely-used suite of analysis and optimization codes that aid aircraft designers during the conceptual design of high-speed civil transport. VizCraft combines visualization and computation, encouraging the designer to think in terms of the overall problem-solving task, not simply using the visualization to view the computation's results. Also, a computing environment developed by Chen et al. [9], that combines particle systems, rigid-body particle dynamics, computational fluid dynamics, rendering, and visualization techniques to simulate physically realistic, complex dust behavior has been shown to be useful in interactive graphics applications for education, entertainment, or training.

\subsection{WBC Computer-based Systems}

In the wood composites industry, mathematical models may be used to describe the pertinent relationships among various manufacturing parameters and final composite properties. The most influential stages of composites production are the mat formation and the hot-pressing processes; therefore, the modeling effort is concentrated on these two areas. However, most of the mathematical models were implemented as command line based programs with standard text input and output, and only a few attempts were made to create more user friendly environments.

A mat formation model is required to establish the critical relationships between the structure of the composite and the dynamic change of certain physical properties during mat 
consolidation. One of the most notable examples of such an approach is the commercially available mat formation software developed by Forintek Canada Corporation. The software, based on Dai's mathematical model [10], incorporates geometric probability theory and simulation techniques for describing characteristics observable on the surface of the flake mat. The basis of the model is an idealized, randomly formed, flake layer network, where the dimensions of the flakes are uniform, and the number of the flakes is limited in a manner that the sum of the areas of the flakes is equal to the area of the layer. Given these assumptions, several properties of the flake network are mathematically definable using probability distributions and random field theory. The composite mat is constructed from these single layers of flakes. The model can calculate the horizontal density distribution of the mat, the flake-to-flake contact area, and the void volume fraction. The commercial software version of the model is only accessible for a fee.

Another mat formation model created by $\mathrm{Lu}$ [24], called WinMat, works as a stand alone application in all Windows operating systems. Besides the major objective of WinMat to generate commands for an industrial robot, which is capable of putting together mat structures from uniform size flakes, several additional features were included in the program. It can create a single or a three-layer OSB structure from flakes either with fixed dimensions or with dimensions described by a normal, uniform, Poisson, or binomial distribution. Additionally, the orientation of the flakes can follow a uniform or a vonMises distribution. Although the interface to the program is easy to use, several aspects of the model are deficient. The density of the flakes is constant, not allowing mats built from mixed wood species. Additionally, the number of the deposited flakes in each layer is controlled by the actual flake number instead of the more realistic cumulative flake weight.

Less computer based than the above, another mathematical modeling approach to characterize the spatial structure of randomly formed wood flake composites is referred to as the edge model [21], [22], [23]. This model subdivides the mat into imaginary flake columns of finite size. The geometrical properties of the columns are determined by experiments and measured at the edge of the mat. Probability density functions are then fit to the data. The columns are reproduced in the simulation model by sampling the fitted probability distributions. Although the edge modeling approach is unique, no attempt was made to include a graphical user interface or advanced result visualization.

Zombori et al. [34] developed a more realistic model of the mat structure, where the mat formation includes the geometry of the wood elements as random variables with certain limitations imposed on the orientation of the elements. Additionally, a density value is assigned to each individual strand, allowing the simulation of the horizontal density distribution of composites made out of mixed species. The mat formation process model was developed based on data obtained from commercial strand measurements and was validated by comparison of the simulated horizontal density distribution to a measured horizontal 
density distribution. None of the previous models could predict the real structure and horizontal density distribution of a commercial, three-layer OSB mat, based on geometry and density measurements of industrial strands.

A hot-pressing model describes the heat and mass transfer during the hot-pressing process of wood-based composite panels. The first of a series of models was published by Humphrey and Bolton et al. [4], [5], [6], followed by a more sophisticated model, including steam injection, by Haselein [15]. This was further improved by Thömen [28], who modeled the internal environment in a continuous hot-press.

All these models had inherent limitations; either they were one-dimensional, or gross simplifications were made about the transfer mechanisms. Additionally, important modeling efforts to date were either not computer based or did not include an integrated environment. The hot-compression model of Zombori [33] allowed a comprehensive two-dimensional description of the heat and mass transfer phenomena during the mat consolidation. All conceivable simultaneous heat and mass transfer mechanisms were considered, together with phase balancing sorption isotherms. By numerically solving the governing partial differential equations, the evolution of moisture and temperature profiles in the vertical midplane of the board could be predicted.

The oriented strandboard mat formation and hot-compression simulation models provided in the PSE WBCSim are a logical continuation of the previously described models. Generally, both of the models implement a wider range of functionalities than any of the mathematical models used in the wood-based composites industry. WBCSim supports these models with intuitive graphical user interfaces, from a large selection of model input variables to sophisticated visualization and optimization techniques.

\subsection{Experiment Management Systems}

The topic of experiment management has become very popular in the research community. Workflow management systems (WFMSs) created at the University of Wisconsin [1] use a database management system (DBMS) to store task descriptions and implement all workflow functionality in modules that run on top of the DBMS. The Desktop Experiment Management Environment (DEME), also called Zoo [18], which comes from the same university, emphasizes generic experiment management technology. Zoo has been used by many domain scientists in fields as diverse as soil sciences to biochemistry, demonstrating that new technology can be continuously transferred among laboratories. At the same time, feedback from installed software can be tested and evaluated in real-life settings that can also affect research directions and decisions. The Site-Specific Systems Simulator for Wireless Communications [31] at VPI uses a subset of XML-based markup languages to support a high performance execution environment, experiment management, and reasoning about model sequences. PYTHIA-II [16] is a modular framework and system that combines a 
general knowledge discovery in database methodology and recommender system technologies to give advice about scientific software/hardware artifacts. It provides all of the facilities needed to set up database schemas for testing suites and associated performance data in order to test suites of software. 


\section{Chapter 3: SIMULATION MODELS}

WBCSim currently supports five simulation models that help wood scientists studying wood-based composite material manufacturing. Each of these models is described next.

\subsection{Rotary Dryer Simulation (RDS)}

The rotary dryer simulation model assists in the design and operation of the most common type of system used for the drying of wood particles [19], [20]. The rotary dryer is an integral part of the processes to manufacture particleboard and strandboard products. It consists of a large, horizontally oriented, rotating drum (typically 3 to $5 \mathrm{~m}$ in diameter and 20 to $30 \mathrm{~m}$ in length). The wet wood particles are mixed directly with hot combustion gases, in a co-current flow pattern, at the inlet to the rotating drum. The gas flow provides the thermal energy for drying, as well as the medium for pneumatic transport of the particles through the length of the drum. Interior lifting flanges serve to agitate and produce a cascade of particles through the hot gases.

The RDS model consists of coupled material and energy balance equations for each segment along the length of the drum. Each drum segment is defined by the cascading pattern of particle travel. The segment, or cycle, begins when a particle drops off a lifting flange and falls to the bottom of the drum. This is followed by travel along the periphery of the drum, where the particle is caught by a lifting flange. The segment ends when the particle attains its maximum angle of repose and tumbles off of the lifting flange. The user must supply the inlet conditions of the hot gases and wet wood particles, as well as the physical dimensions of the drum and lifting flanges, flow rates, and thermal loss factor for the dryer. The RDS model predicts the particle moisture content, temperature, gas composition, and energy consumption.

\subsection{Radio-Frequency Pressing (RFP)}

The radio-frequency pressing model [26] was developed to simulate the consolidation of wood veneer into a laminated composite using high frequency energy. The energy needed for cure of the thermosetting adhesive is supplied by a high-frequency electric field. Radiofrequency pressing is commonly used for thick composites and for nonplanar laminated composites. The model may be used to help design alternative pressing schedules.

The RFP model consists of a collection of nonlinear PDEs that describe the heat and mass transfer within the veneer layers. The primary variables are temperature and moisture content. The moisture content is further divided into three phases: bound water, liquid water, and water vapor. These water phases must satisfy a criterion of local thermodynamic equilibrium as represented by a nonlinear algebraic equation. The model is one-dimensional, with a fixed resistance to heat and mass flux at the boundary. The RFP model predicts the time-dependent temperature and moisture content profiles in the veneer layers, as well 
as the extent of adhesive cure. Among the user-supplied input data are the initial density, thickness, moisture content, and temperature of the veneer, as well as the electric field strength.

\subsection{Oriented Strandboard Mat Formation (OSB)}

The mat formation model creates a three-dimensional spatial structure of a layered wood-based composite (e.g., oriented strandboard and waferboard); it also calculates certain mat properties by superimposing a mesh on the mat structure. The model is based on a Monte Carlo simulation technique. It is assumed that the dimensions of the constituents (e.g., strands) of the mat follow certain probability distribution functions. The parameters of the probability distribution functions have to be determined on a subsample by measurement. The user inputs the number of layers. The total number of strands is determined by specifying that number, or specifying the weight, volume, or area of each layer. The probability distribution functions of the length, width, thickness, and density of the strands are given by the user.

Output from the mat formation model includes the horizontal density distribution, proportion of void space, and potential bonded surface area. Output data is available in a numerical format and two- or three-dimensional visualizations. The output from the mat formation model is required for the hot compression model.

\subsection{Hot Compression (HC)}

The hot compression model simulates the mat consolidation and adhesive cure that occurs during industrial hot-pressing of wood-based panels. The model is based on fundamental engineering principles and uses the output from the mat formation model to establish the starting spatial structure of the mat. Six primary variables are considered: mat density, air content, vapor content, bound water content, and temperature within the mat, and the extent of the cure of the adhesive system characterized by the cure index. The heat is transported by conduction and bulk flow, while the water phases are transported by bulk flow and diffusion. A nonlinear viscoelastic relationship was used to describe the compression behavior of the mat. This relationship separates the geometric nonlinear response of the cellular structure of the wood elements from the linear viscoelastic response of the wood cell wall polymers. The behavior of the cellular structure is modeled with a modified Hooke's Law. The viscoelastic properties of the flakes are described by the time-temperature-moisture equivalence principle of polymers.

The resulting differential-algebraic system of equations is solved by a semicontinuous finite difference method. The spatial derivatives of the conduction terms are discretized according to a central difference scheme, while the spatial derivatives of the bulk flow terms are discretized according to an upwind scheme. The resulting ordinary differential equations 
(ODEs) in the time variable are solved by DDASSL, a freely available differential-algebraic system solver. The model predicts temperature, moisture content, partial air and vapor pressures, total pressure, relative humidity, extent of adhesive cure, and the spatial density profiles within the mat. A set of three-dimensional graphical profiles illustrate the evolution of these variables with time, in the thickness and width dimensions of the mat. The model assists users in the understanding of the interacting mechanisms involved in a complex production process. The model may also be used to optimize the hot-pressing parameters for improved quality of wood-based panel products, while minimizing processing cost.

\subsection{Composite Material Analysis (CMA)}

The composite material analysis model was developed to assess the stress and strain behavior and strength properties of laminated materials (e.g., plywood and fiber-reinforced composites). The user defines the layer sequence of the laminate. The graphical user interface was designed to allow easy specification of the material, thickness, and orientation at each layer. The mechanical and failure properties of the layer materials may be selected from a predefined list. The model can perform design and analysis functions, where the user either defines the loading condition, or defines the deformation.

The calculations are based on the classical lamination theory (CLT) and the Tsai-Wu failure criteria. The "Design" and "Analysis" models calculate the induced stresses and strains in the primary laminate $(x, y)$ and principal fiber $(1,2)$ directions within each layer based on the CLT, and check for the integrity of the layers of the laminate by the Tsai-Wu failure criteria. In the design mode, the model calculates the stresses and strains caused by the combination of different loading conditions, such as tension, moment, torque, or shear. In the analysis mode, the normal and shear stresses, together with the strains and curvatures induced by a user-defined deformed shape, are calculated. The model predicts the tensile strength, bending strength, and shear strength of the composite material. 


\section{Chapter 4: SIMULATION MODEL USER INTERFACES}

WBCSim is Web-based; therefore, its user interface is composed of a Web browser and Java applets. A user launches the WBCSim Web page from a browser window, and then invokes applets from the Web page. The very first applet allows the selection of a simulation model. From that point on, all user interaction with the system is via applets. There are some common features among all the model interfaces (see Figure. 4.1). At the top left corner of a model interface, there is a "Usage Instructions" button, whose pop-out window explains the model behavior at an abstract level, and also gives general directions about how to use the model. The middle region of each model interface is model-dependent, allowing a user to specify simulation parameters in any number of text boxes, drop down lists, radio buttons, or plain buttons, which possibly open pop-out windows for editing even more simulation parameters. Near the bottom of the interface, there is a long text box where a user can describe the simulation; this description is saved when the simulation data is saved. At the very bottom of an interface, there is a row of buttons, which control model-level actions for a simulation. Among those buttons are the four: "Retrieve Problem", "Run Simulation", "Store Problem", and "Dismiss". The "Store Problem" button is used to store the current set of input values (along with the simulation description text), which can be retrieved later using the "Retrieve Problem" button. (The user interface related to the EM component are described in Chapter 8.) Clicking on the "Run Simulation" button fires input values defined in the interface through a telnet connection to the WBCSim server, which in turn calls a UNIX or Perl script to execute compiled FORTRAN code, and additional optimization or visualization codes. (The details of this communication process are described in the Chapter 5.) The "Dismiss" button dismisses the interface without saving any input. Other than these four main control buttons, there are some other buttons varying from model to model that may appear in the same row, such as the "Simulation Constants" button (lists some simulation parameters not accessible by the user), and the "Set Default" button (sets all the model variables to a predefined set of values). The results from running a simulation model are usually available in both textual (normally tables of numbers) and graphical (VRML files, GIF files) forms, and displayable in browser windows.

The RDS (Figure. 4.1) and RFP models have the simplest user interfaces in WBCSim. The user simply enters values for various input parameters through text boxes, then runs the simulation. The CMA model has evolved. Depending on the context, "model" refers to the computer simulation code itself, or the interface to that code. The previous CMA model [14] supported five calculations: "analysis", "design", "tensile strength", "bending strength", and "shear strength". When the user selected any of the five, the user was locked to that calculation and could not switch from one calculation to another without returning to the beginning. Also, in each calculation interface, many parameters were fixed or 


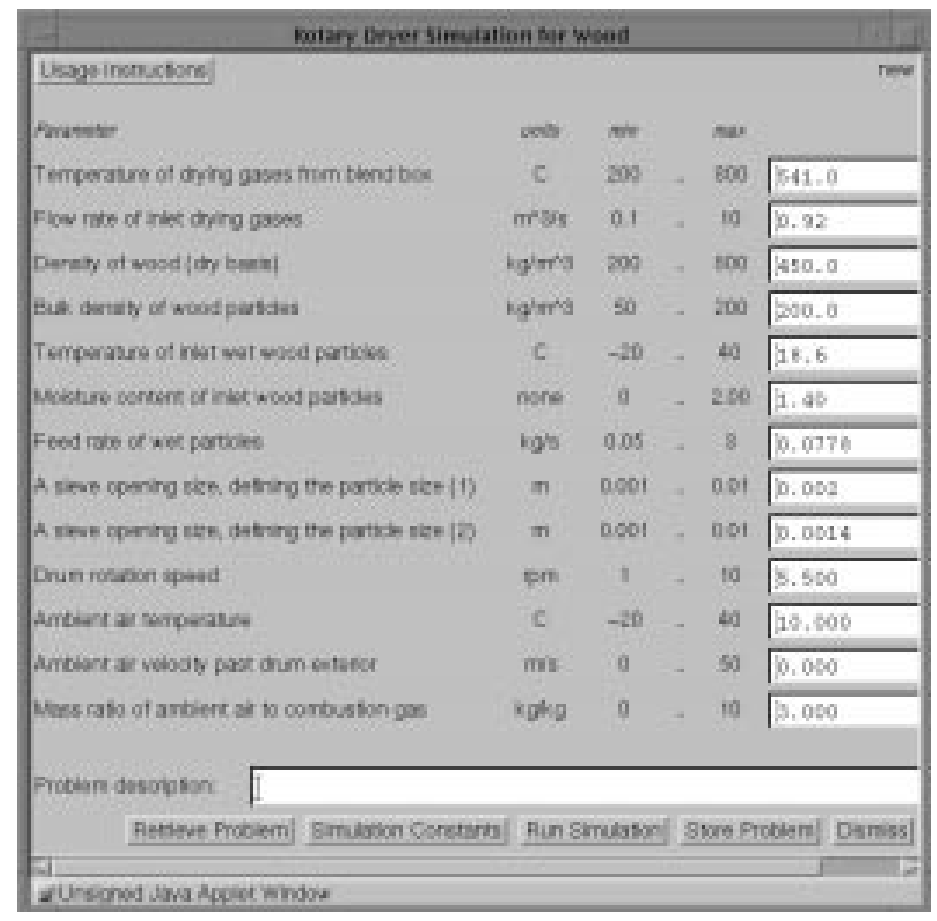

Figure 4.1. The RDS model user interface.

assumed, whereas the current CMA model interface provides text boxes for those previously fixed parameters. The current CMA model has a new calculation "general strength", and integrates all the calculations into one interface, allowing the user to select a dynamically loaded calculation from a drop down list. The current CMA model also includes two sets of defaults, structure and loading (both in drop down lists). This new integrated interface allows the user to cross-reference different sets of defaults by setting one structure parameter set and then running any calculation with any loadings.

The OSB model (Figure. 4.2) is similar to the CMA model with rows of input data representing layers of the wood composite. The user activates a layer by selecting the leftmost checkbox for that layer. Also as in the CMA model, the OSB model provides a set of defaults rather than a single default like most of the other models. However, the rest of the OSB model is more complex than that of CMA, since it involves many pop-out windows when the user chooses an item from a drop down list or clicks on a button. For example, selecting the "Empirical" distribution in the "Length Distribution" drop down list, a pop-out window containing forty-two text boxes appears, allowing the user to define the number of intervals and each interval's starting and ending points. The OSB interface can also save the values defined in pop-out windows, which means those pop-out windows can be closed and then opened again for editing.

The HC model (Figure. 4.3) interface differs from the other models' interfaces by dividing the simulation parameters into three groups partitioned into columns: (1) model input, (2) 


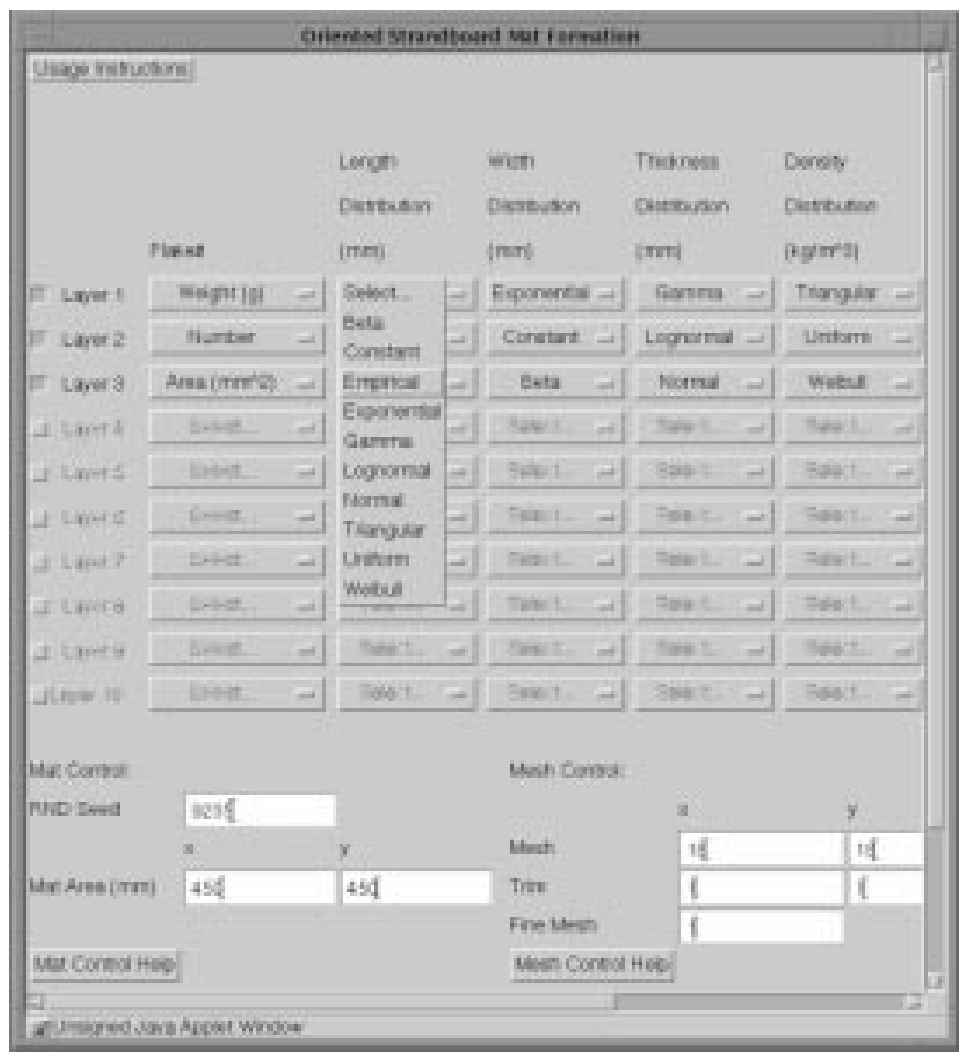

Figure 4.2. The OSB model user interface.

model execution specification, and (3) model output. The first column contains buttons for specifying the parameters related to material, initial condition, boundary condition, adhesive cure, and press schedule. The second column defines the HC model execution by specifying mat transport properties, boundary transport properties, and compression properties. The last column specifies the output such as color or black and white, the time interval for generating frames for animation, and other parameters determining which data/image files are generated. 


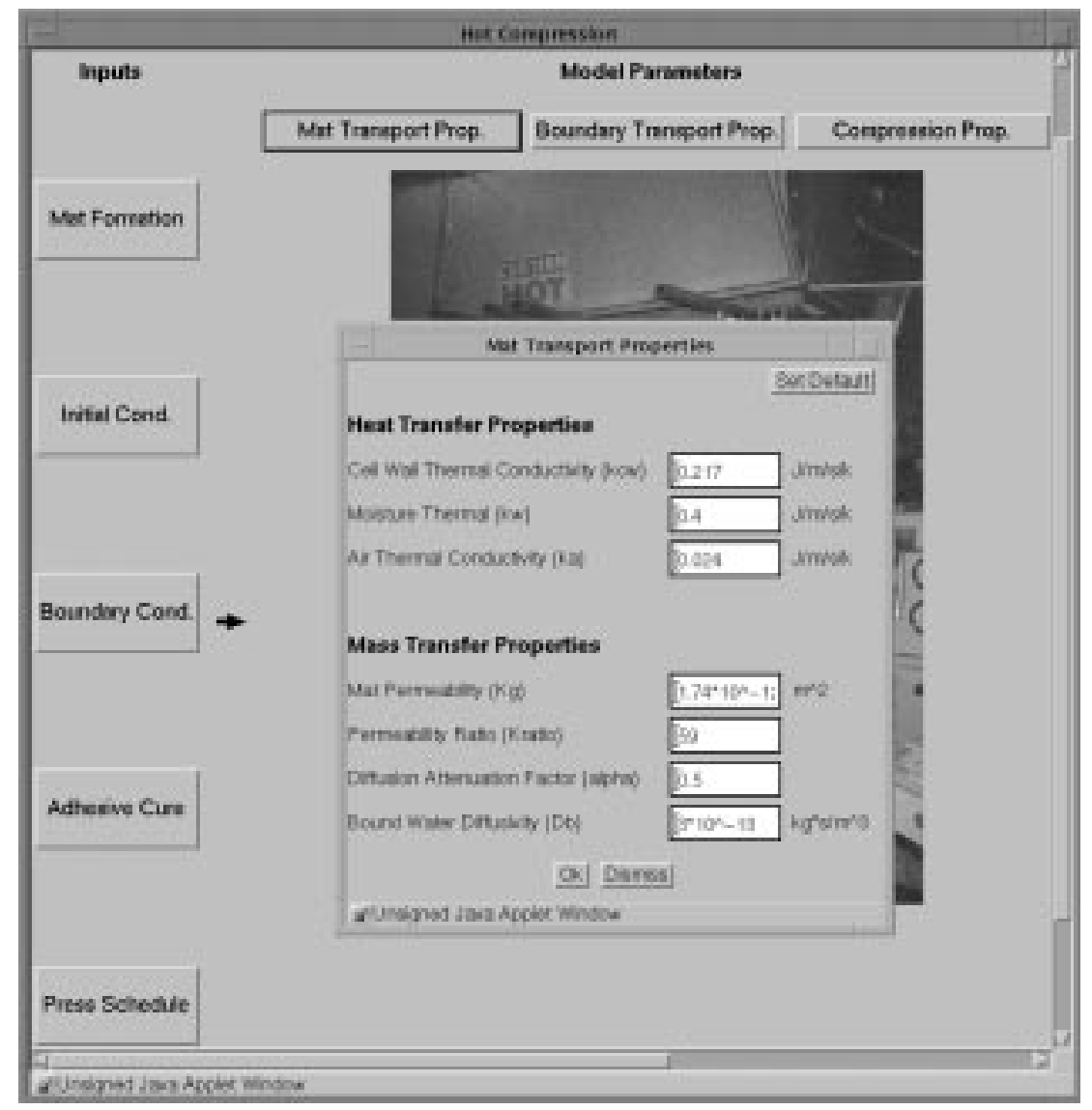

Figure 4.3. The HC model user interface. 


\section{Chapter 5: WBCSIM ARCHITECTURE}

The current software architecture of WBCSim follows the three-tier model described by Goel et al. [14], for which the tiers are (1) the client layer-user interface, (2) the server layer - telnet server and a custom shell, and (3) the developer layer-legacy simulation codes and various optimization and visualization tools running on the server. These layers are shown in Figure. 5.1. (The elements related to the EM component are discussed in Chapter 8.)

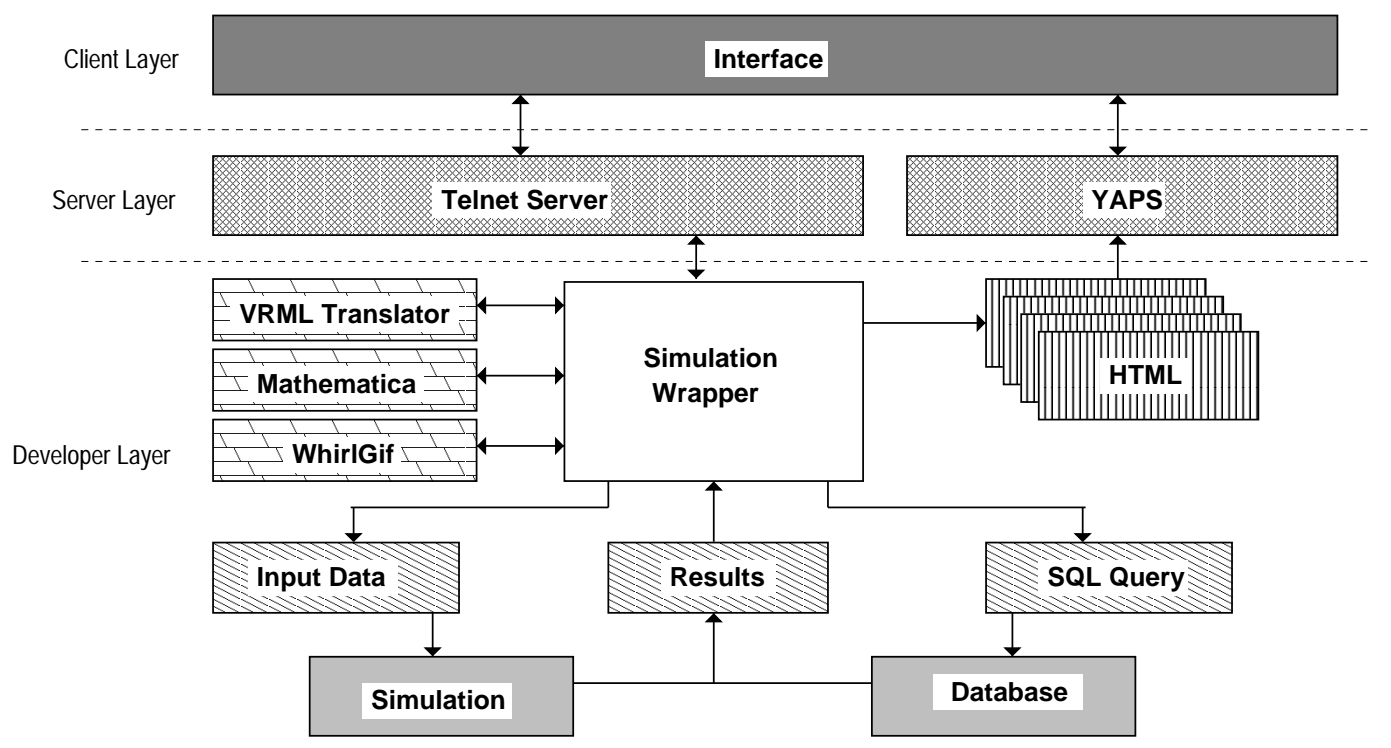

Figure 5.1. WBCSim architecture overview.

\subsection{Client Layer}

The client layer is the only layer visible to end-users and typically the only layer running on the local machine. Besides the user interfaces described in Chapter 4, the client layer also contains viewers for one of the visualization tools - the VRML Translator. WBCSim requires a VRML 2.0 viewer for the RFP model. The VRML viewer serves as a plug-in to the Web browsers.

The client layer also handles the communication with the server layer. After the user enters all the necessary parameters and triggers the "Run Simulation" button, the client sends those parameters along with a request for executing the corresponding simulation to the server layer via a telnet connection. When the simulation terminates, the client takes the URLs returned by the server layer, and directs them into the user's browser. Of course, the client may also send requests for save and retrieve operations.

The telnet connection method replaces the old Javamatic server and socket communication paradigm described in [14], and facilitates setting up WBCSim guest accounts on the 
server machine that do not require full account privileges. The guest account and normal user accounts (for commercial, paying users) permit users to save and track their different simulation runs, and protect their data from being removed or overwritten by others. The client side of this telnet connection manages the pool of connections and provides a means for executing remote commands on the server.

\subsection{Server Layer}

By separating the legacy simulation codes from the user interface, the server layer functions as the key to how WBCSim can run a text-only application from a Web browser. The server layer consists of two components: a telnet server and a custom shell to facilitate server-client communication.

The telnet server is not a replacement for a standard telnet server, implementing only enough of the telnet protocol to work with the WBCSim telnet client. The telnet server supports guest and regular logins and all the operations provided by the previous Javamatic server, which could direct execution of multiple simulations and accept multiple requests from the client concurrently [14].

Yet Another PSE Shell (YAPS) is a simple Perl script that the client invokes when it logs in via the telnet connection. Therefore, the client talks to this shell instead of the UNIX login shell of the account. Among the commands that YAPS supports, the following four are most important.

(1) The store command is used to permanently store a problem (input parameters, text description, simulation output) in the database on the server. Notice that it is possible to store problem parameters without running the simulation, in which case there is no simulation output.

(2) The remove command removes one or more stored problems. It actually removes entries from the database, so the results are irreversible.

(3) The load command retrieves all stored problems from the database. This command prints a collection of problem IDs (each problem definition or simulation run has a unique problem ID), parameters, descriptions, and URLs, which could be picked up by the telnet client.

(4) The clean command deletes temporary files. Normally cleanup is requested when the "simulation results" window is dismissed. However, cleanup requests are only executed if there is an idle connection. If no idle connection exists, cleanup requests are queued until a connection becomes available. The goal of this policy is to clean up only when doing so incurs no extra connection. Making an extra connection involves a lengthy bootstrap process and possibly passing the user password again.

There is no command to run a simulation because the simulation wrapper can be invoked directly by YAPS based on the requests coming from the telnet client. 


\subsection{Developer Layer}

As its name suggests, the developer layer consists of legacy programs created by researchers to model wood-based composite materials and manufacturing processes. These legacy programs are the heart of WBCSim. In general, WBCSim supports legacy programs written in any programming language as long as the program takes its input parameters from UNIX stdin. The input parameters can be a few numbers or a large data file. In particular, WBCSim supports five FORTRAN 77 and Fortran 90 simulation programs, corresponding to the five models described in Chapter 3.

While each Fortran program has its own input format (stdin could be redirected to a file), the server layer communicates data with the developer layer via strings of parameters separated by white space (spaces, tabs, newlines). In order to cope with this string format, each legacy program is "wrapped" with a customized Perl script. The script receives this string of parameters from the server, and converts those parameters into an appropriate format for the Fortran program. Then the script calls the legacy program into action, feeding it the input, invoking any required optimization and visualization tools, packing all Fortran output in HTML files, and passing their URLs, first to the server layer, and then to the client layer. With this architecture, the developer layer is independent of the other layers, which makes the process of designing, and integrating new simulation codes relatively easy.

The developer layer also includes optimization and visualization tools, which are described in the next chapter. 


\section{Chapter 6: OPTIMIZATION AND VISUALIZATION}

As mentioned in Chapter 5, there are optimization and visualization tools residing in the developer layer to maximize the simulation's value to the user. Here optimization and visualization are described, respectively.

\subsection{Optimization}

Scientists typically use a PSE like WBCSim iteratively with some design or system behavior goal in mind. Their exploration is by trial and error, guided by intuition that improves as more computational experiments are performed. Mathematical optimization is a powerful tool for automating this tedious search process. Currently the optimization tool provided with WBCSim is the program DOT (Design Optimization Tool) [29], based on sequential quadratic programming and the method of feasible directions. DOT is a robust sophisticated FORTRAN 77 subroutine for nonlinear constrained optimization, and is widely used in engineering.

WBCSim supports two models, RDS and RFP, that are linked to DOT. Each has a different interface for optimization than for analysis. Comparing the interface without the optimization option to the one with optimization, the former provides only one text box for every simulation parameter, while the latter provides three, one for specifying the initial value for that parameter, one for specifying the maximum value, and one for specifying the minimum value. The optimization interface also allows the user to specify which parameters are fixed and which are to be varied. The optimization interface also supplies a number of predefined objective functions (typically computed physical quantities, such as temperature or pressure), for which the user can specify either maximization or minimization. The optimization interfaces necessarily provide parameters to control the convergence of the optimizer, such as "Maximum absolute change in the objective function", "Maximum relative change in the objective function", "Relative finite difference step for gradients", and "Maximum number of iterations".

\subsection{Visualization}

In principle, WBCSim supports the addition of any visualization tools to convert simulation output to a format meaningful to the user. However, it does require the user to have the corresponding viewer for the format of that visualization result installed and accessible from the Web browser. An example is the VRML viewer described in Chapter 5.2.

WBCSim currently uses three visualization tools: VRML [2], Mathematica [32], and the UNIX utility WhirlGif. With a VRML viewer, the output converted to VRML can be viewed from various directions in the three-dimensional viewspace. Mathematica is only used to generate static two or three-dimensional graphs of simulation results. WhirlGif is 


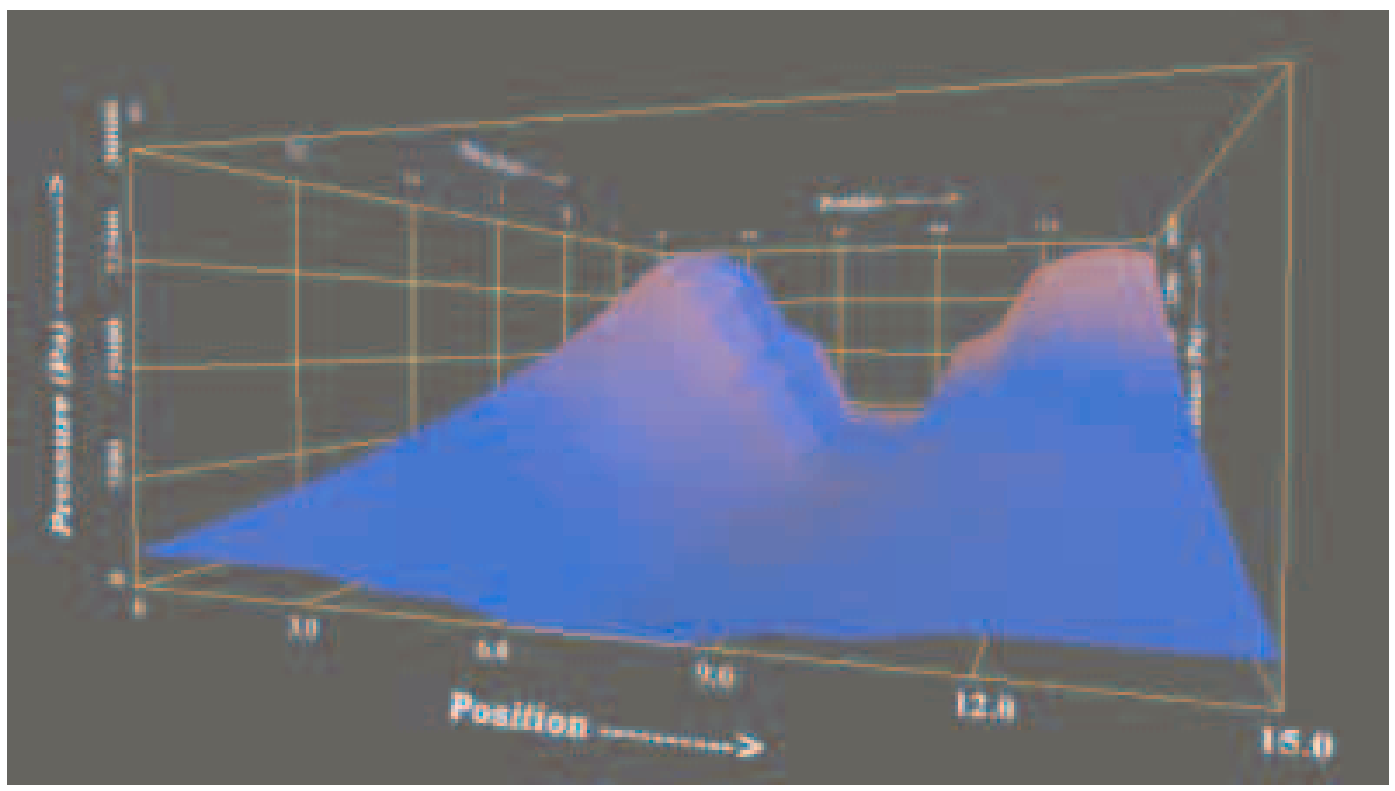

Figure 6.1. VRML pressure graph showing the variation of pressure with increasing distance from the laminate surface and with time (the receding axis)

a GIF to animation converter, which simply picks up the GIF format frames generated by Mathematica and converts them into an animation file, also in GIF format.

VRML was chosen to interactively visualize some WBCSim simulation output, because VRML is a well-accepted standard for three-dimensional visualization, available on many platforms, easy to use, and easily generated. A custom VRML translator was built to provide better control over the description of the three-dimensional output than third party translators could provide. WBCSim generates VRML code for the RFP model output. In the RFP model, the output data is a matrix of numbers where each row contains two independent variables and the corresponding dependent variable. This three-dimensional data is conveniently modeled as a VRML ElevationGrid. Each row in the matrix can be represented either as a point or a square that is colored based on its height, where blue denotes the lowest point on the grid, red the highest, and the rest are colored by linearly interpolating between blue and red (HSB) hues, as shown in Figure. 6.1.

Mathematica was chosen to generate static two or three-dimensional graphs of WBCSim simulation results, because Mathematica has sophisticated graphics tools, and is commonly used by wood scientists for graphic representations. When Mathematica was first used in conjunction with WBCSim, the Mathematica commands were hard coded (in Perl) in the wrapper. Every time a simulation was invoked that required Mathematica's services, the wrapper would write the Mathematica commands into a text file embedded with the dynamically generated filenames for the GIF output files (using the simulation ID) . Then, the wrapper would give this file as input to Mathematica, which would generate the GIF 
files accordingly. However, as more complex simulations such as CMA were added to the system, the Mathematica input file became large and unwieldy. Compounding this size problem was the fact that every time the Mathematica code changed, the Perl wrapper also had to be changed. The current WBCSim approach keeps the Mathematica code as a blueprint external to the wrapper. Then in every simulation run, the wrapper makes a copy of that blueprint code, uses sed to insert the dynamic graphics output filenames, and then feeds it directly to the Mathematica kernel. Figure. 6.2 (are explained in Chapter 9) is an image file generated by Mathematica from the OSB model.

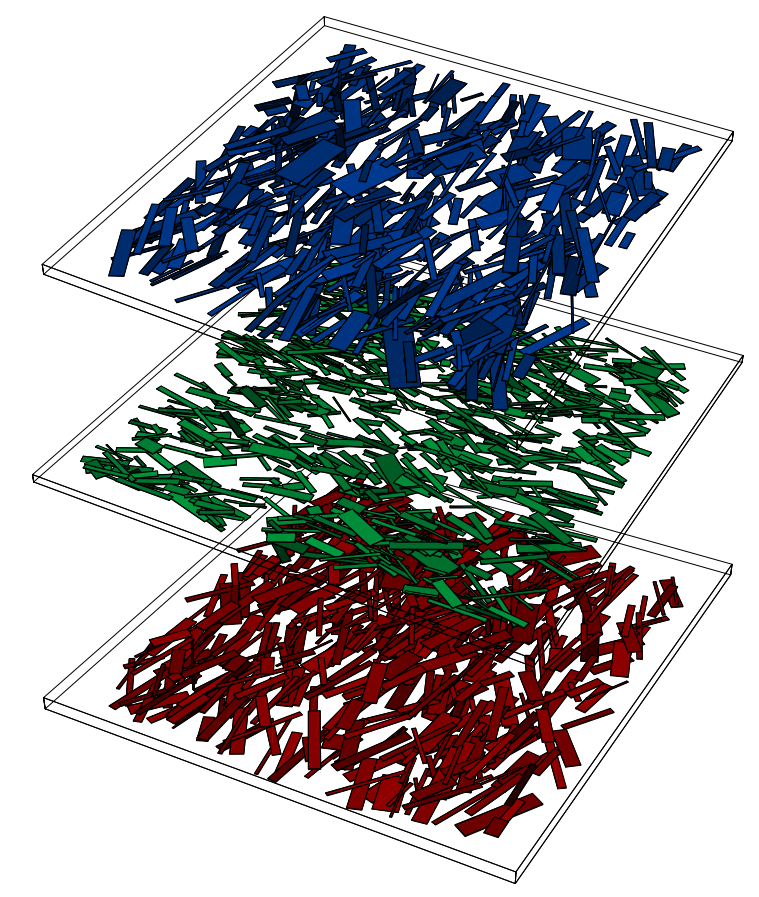

Figure 6.2. Visualization of the three-layer random flake mat: the area of the mat is $450 \times 450 \mathrm{~mm}$. Only a fraction of the flakes are shown, and the shading designates the density of the flakes.

Since some simulation results, such as the CMA model's stress and strain distributions, are time series, WBCSim also provides time animation of those results by using WhirlGif. WhirlGif is a small UNIX utility for generating a single animated GIF image file from a sequence of GIF image files. The reason Mathematica is not used for generating those animation files is that the time to generate an animation in Mathematica is significantly longer than the time by using WhirlGif. Because the number of frames may change from one simulation run to another, the wrapper reads the number of frames from a data file. Then the wrapper constructs a command like "WhirlGif -loop 1000 frame1.gif frame2.gif 
frame3.gif frame4.gif ...> animation.gif", where frame*.gif are the frame images generated by Mathematica. Then this WhirlGif command is fired off to the UNIX shell by the wrapper to generate the animation file. By embedding each frame into a HTML file, another option is also provided whereby the user can use hyperlinks such as "Next" and "Previous" to view the frames one at a time. 


\section{Chapter 7: RATIONALE FOR EXPERIMENT MANAGEMENT}

Before the experiment management component was added, WBCSim had a file-based save and retrieve system that operated as follows. Upon saving, the input parameters were packed into a file with a filename identified by the current run ID. This file, along with the possible output files (data files generated by the FORTRAN code, pictures generated by the Mathematica or VRML translator, and HTML files generated by the scripts), was stored in a permanent directory (depending on the login account). An optional description of the simulation was also stored. Upon retrieving the simulation, a user chose to either load the input parameters in the proper model interface or load the stored simulation results.

This approach is not sufficient as WBCSim evolves from being experiment-oriented to being manufacturer-oriented. Manufacturers like to see how the product properties could be optimized while the profit is maximized. However, any given simulation run is just an evaluation of a single point in a multidimensional space, and optimization can only search this space for an optimal point with respect to an objective function [27]. While WBCSim supports automated optimization, often it is not possible to precisely articulate an objective function, and there may be multiple conflicting objectives. Furthermore, design trends and data patterns are unlikely to be revealed by a few isolated runs or by automatic optimization. What is required is that the results of simulation runs be stored automatically in a systematic way. This approach would permit annotating the parameters that define a run as well as its results, and would further allow searching, comparison, and other data mining of a database of numerical experiments.

Toward this end, a special tailored experiment management component is being added to WBCSim, which consists of customized user interfaces, server scripts, and an open source database management system (DBMS) - Postgres. The details of this component are explained in Chapter 8. This experiment management component not only supports all of the previous features from the file-based save and retrieve system, it also significantly increases WBCSim user productivity and usability in the following ways.

(1) The new user interface allows a user to filter the stored simulation runs by their description or date.

(2) The new user interface provides customized comparison functions that can compare multiple simulation runs.

(3) The server script searches the database for each simulation run. If the simulation has been run before and saved, the script retrieves the simulation results from the database rather than run the FORTRAN program, which normally requires a longer execution time.

(4) The database stores simulation inputs and outputs. Only distinct inputs and outputs are saved. In case there is a duplicate simulation run (with a distinctive annotation), its inputs and outputs are not stored but will point to those from the previously stored simulation run. 


\section{Chapter 8: EXPERIMENT MANAGEMENT COMPONENT ARCHITECTURE}

Chapter 5 explained the WBCSim three-tier model. Here, only the elements related to the experiment management component are discussed.

\subsection{Client Layer}

Chapter 4 described how all the models have a row of buttons at the very bottom of the interface (shown in Figure. 4.1) that control model-level actions for a simulation. Among those buttons are two, "Store Problem" and "Retrieve Problem", which are related to the experiment management component. When the "Store Problem" button is triggered, the current set of input values (along with the simulation description, which can be specified in the long text box near the bottom of the interface) is sent to the server and stored. The interface are updated with a run ID at the upper right-hand corner to indicate that the save occurred. The "Retrieve Problem" button is used to retrieve stored simulation runs.

Upon activation of the "Retrieve Problem" button, a window (shown in Figure. 8.1) will open with a list of all the simulation runs for this particular model stored in the database. This window is the portal for a user to access the stored simulation runs. At the top of this window, there is some text describing the use of this window. There are also a set of dropdown lists, and text fields and buttons, which allow the user to filter the simulation runs displayed. By selecting either "Description" or "Date" from the "Options" dropdown list, the user can narrow the filter process to either field. Then the user can input a regular expression (in UNIX grep style) in the text field, and press the "Filter" button to execute the filter process, which will reload this window with the simulation runs that meet the filter criteria. The next portion of the window is a scrollbar panel with all of the simulation runs listed. Each simulation takes a row, which has a "Show Input" button, "Show Output" button, a check box, the date that it was stored, and its description. The "Show Input" button launches the model interface with the set of stored input values. The user can make changes to those parameters, and run the simulation again. Clicking on the "Show Results" button opens another window with buttons that point to the simulation results, displayable in browser windows. The checkbox allows the user to either delete the simulation run from the database (by clicking the "Remove" button at the very bottom of the window), or apply the comparison functions (by selecting an item from the "Compare Marked Runs" dropdown list). Each model of WBCSim has its own comparison functions. For example, the OSB model has three, which allow the user to compare "Void Fractions/Contact Area", "Density", and "Coefficient of Variation" among the simulation runs marked.

If "Void Fractions/Contact Area" is selected from the "Compare Marked Runs" dropdown list, a window (shown in Figure. 8.2) opens with more options that allow comparisons

among "space/mat", "lumen/mat", "void/mat", "lumen/flake", and "contact area". The user can also specify the interval of the $\mathrm{Y}$ axis in order to narrow the comparison.

Depending on the choices made in the detail comparison window, the user gets a result window, which has links pointing to the results that can be displayed in a browser window. 


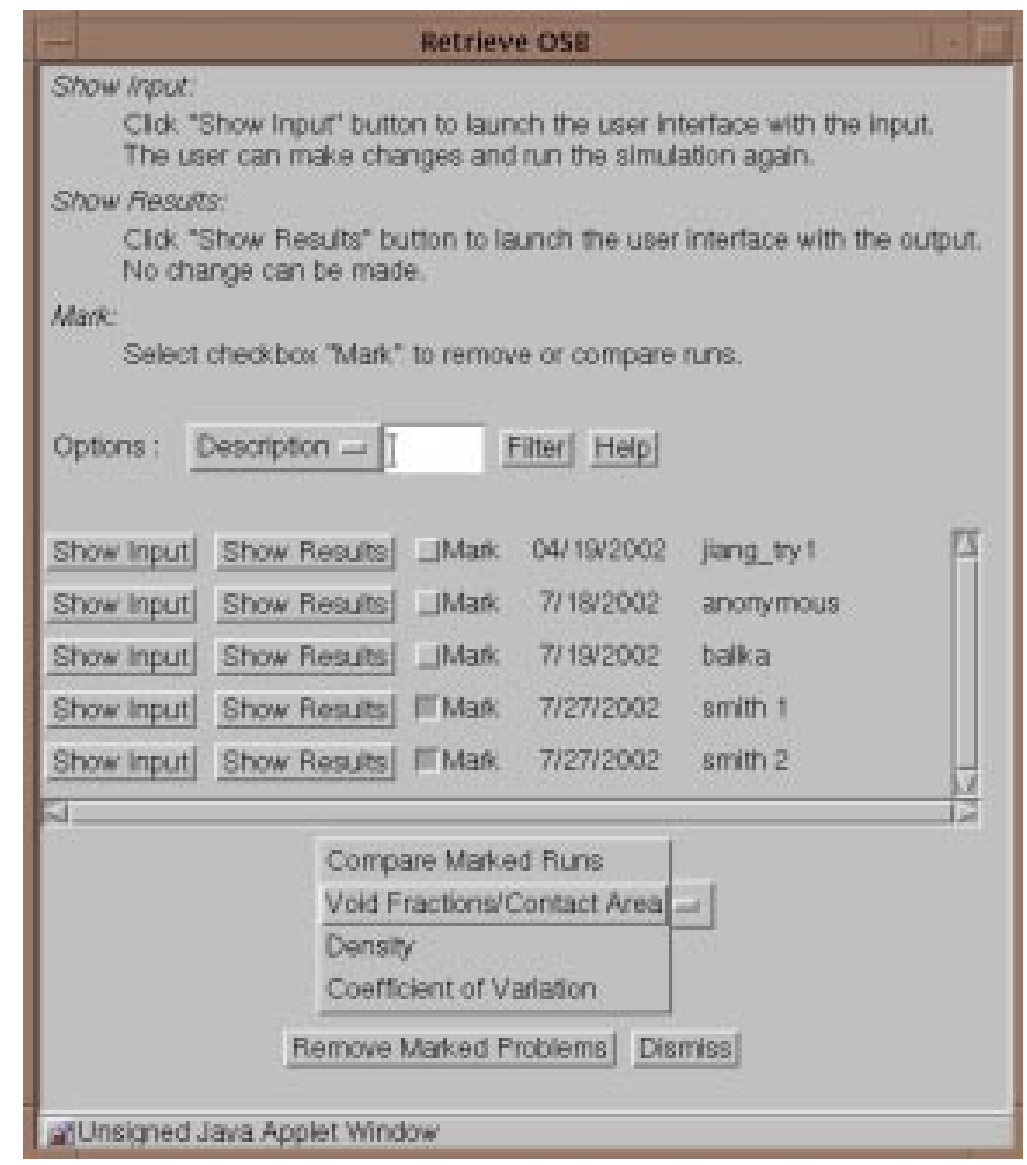

Figure 8.1. The OSB retrieval user interface.

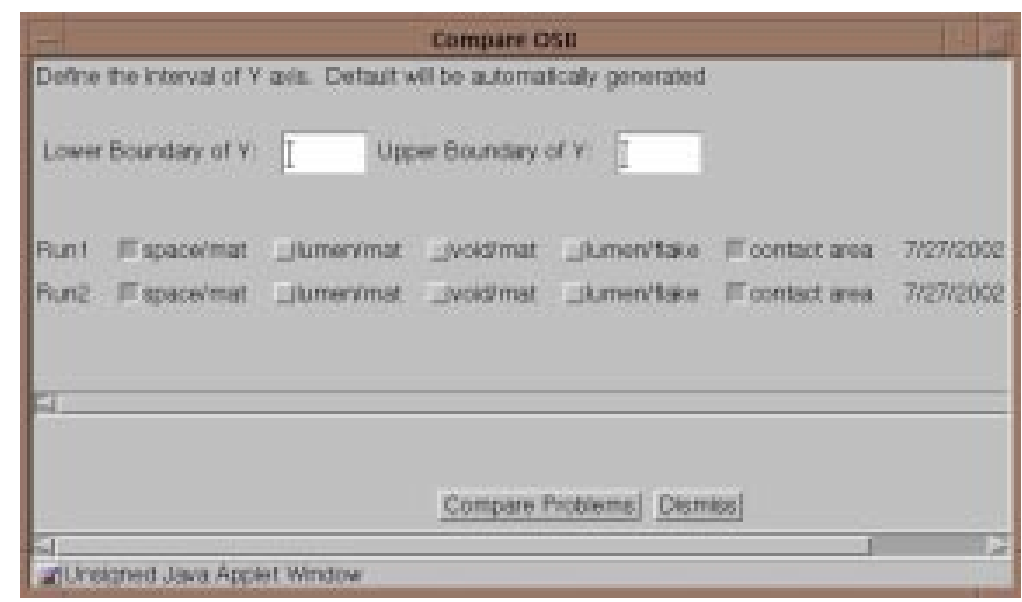

Figure 8.2. The OSB Void Fractions/Contact Area comparison detail user interface.

\subsection{Server Layer}

Chapter 5.2 described the common commands supported by YAPS. Those commands 
were previously working with the file system, but are now calling the corresponding save and retrieve wrappers to interact with the database. These save and retrieve wrappers are explained next.

\subsection{Developer Layer}

With the addition of the experiment management component, the simulation wrapper (described in Chapter 5) performs one more operation before it calls the Fortran program to execute the simulation. The simulation wrapper checks if a simulation with the current set of inputs has already been executed. If the simulation was executed before and the results were saved, the wrapper constructs the output data files from the database instead of running the Fortran program. The wrapper then feeds the output data files into any required optimization and visualization tools. This operation is transparent to the user; it can significantly reduce the response run time if the output is found in the database.

There are other wrappers associated with the save, retrieve, and compare functions from the user interface at the client layer. Like the simulation wrapper, these wrappers receive data from the server layer, and convert those input values into an appropriate format (SQL queries typically) for the database. Moreover, they also perform some extra checks in order to optimize database usage and storage. For example, the save wrapper checks if the current set of values is already saved. If that is the case, only a pointer to that set of values is saved. If the current set of the input that the user is saving is matched with the previous stored inputs whose output was not saved, the wrapper automatically updates those previously stored inputs in order to point to the current set of output values.

Postgres is the database system being used. Postgres is a sophisticated object-relational database management system (DBMS) that supports almost all SQL constructs, including subselects, transactions, and user-defined types and functions. Moreover, it is open source. In this architecture, the wrappers communicate with Postgres by Pgsql-per15, which is an interface between Perl version 5 and Postgres. This interface uses the Perl version 5 application programming interface for $\mathrm{C}$ extensions, which calls the Postgres programmer's interface LIBPQ. 


\section{Chapter 9: SIMULATION SCENARIO}

Describing a typical usage scenario of WBCSim is instructive. Consider research into the properties of oriented strand board products, which would use the OSB model. First, a scientist samples a subset of the face and core layer flakes from an industrial production line. The scientist measures the geometry and the weight of each flake. The flake property data sets are then used to estimate the statistical probability density functions of the flake properties. Then, the scientist opens a browser window and launches WBCSim. The guest account is the default login option, which stores all the input and output data in a temporary directory that is purged periodically. The scientist selects the OSB model from a dropdown list of all the available models, and launches the OSB model interface (shown in Figure. 4.2). By specifying the probability density functions of the flake properties obtained offline earlier, the scientist can create a statistically valid model instance of the spatial structure of multiple layers of flakes, each with various parameters defining the number of flakes, length distribution, width distribution, thickness distribution, density distribution, orientation, flake color, and number of flakes shown. The latter two are solely for visualization.

Next, the input parameters (Boolean, numeric, or alphanumeric) are sent to the WBCSim server as a long string (parameters are separated by white space) via a telnet connection. Then, the OSB wrapper converts this string into a SQL query, and checks if this set of input parameters is stored in the database.

If the output does not exist in the database, the OSB wrapper converts this input string into a data file designated for the OSB Fortran 90 simulation program. Since the OSB simulation code has its own text-based user interface taking input from stdin, a temporary file is generated to contain all of the appropriate commands. Stdin is then redirected to this temporary data file for the simulation. The OSB wrapper then calls the OSB simulation code with this temporary file as stdin along with the properly formatted data file. When the simulation code is executing, the OSB wrapper monitors to the simulation output stream for strings indicating execution milestones. The OSB wrapper uses the standard error stream for sending these messages to the client, because Java buffers the standard output stream until the process terminates. The contents of the standard error stream are sent immediately. The OSB wrapper also sends other messages (such as when visualization or optimization tools are invoked) to the client, displaying the simulation status for the scientist. However, due to network delay, a group of messages generated at different times can arrive at the client at the same time, in which case the client only displays the latest message and discards any old ones.

If the output exists in the database, the OSB wrapper extracts the outputs from the database and places them into data files in the same format as they would have been written by the OSB Fortran 90 simulation code. 
When those output data files are ready (no matter whether they come from the Fortran 90 simulation program or the database), the OSB wrapper calls Mathematica to read those data files and generate plots with various Mathematica commands such as ListContourPlot, ListPlot3D, MultipleListPlot, and Graphics3D. Mathematica also converts these internal graphics data structures into GIF format so that they can be viewed in a browser. Figure. 6.2 shows a three-dimensional visualization of a three-layer random flake mat created with Graphics3D by Mathematica. Finally, the OSB wrapper embeds these GIF files in HTML files and returns the URLs of these HTML files to the client.

Upon closing the interface windows, the scientist has a choice of storing this particular run's input values and results, or discarding this run (the default). If the scientist chooses to store this run, he enters a description such as "Smith 2" and presses the "Store Problem" button. The entire interface is then updated with the run ID to indicate that the save occurred. To further compare this run with any previous runs, the scientist can click the "Retrieve Problem" button to launch the retrieve problem interface (shown in Figure. 4.1). The scientist can use the filter (described in Chapter 8) to narrow the list of simulation runs displayed in the scrollbar panel. By marking two simulation runs, for example, "Smith 2" and "Smith 1," the scientist can compare their "Void Fractions/Contact Area" by selecting that item from the "Compare Marked Runs" dropdown list. The comparison detail window (shown in Figure. 8.2) allows the scientist to choose among "space/mat ", "lumen/mat", "void/mat", "lumen/flake", and "contact area" to view the comparison result. Figure. 9.1 shows the comparison result created with MultipleListPlot by Mathematica. The scientist can go back to the comparison detail window to make other selections or change the $\mathrm{Y}$ axis interval to get different results or views. 


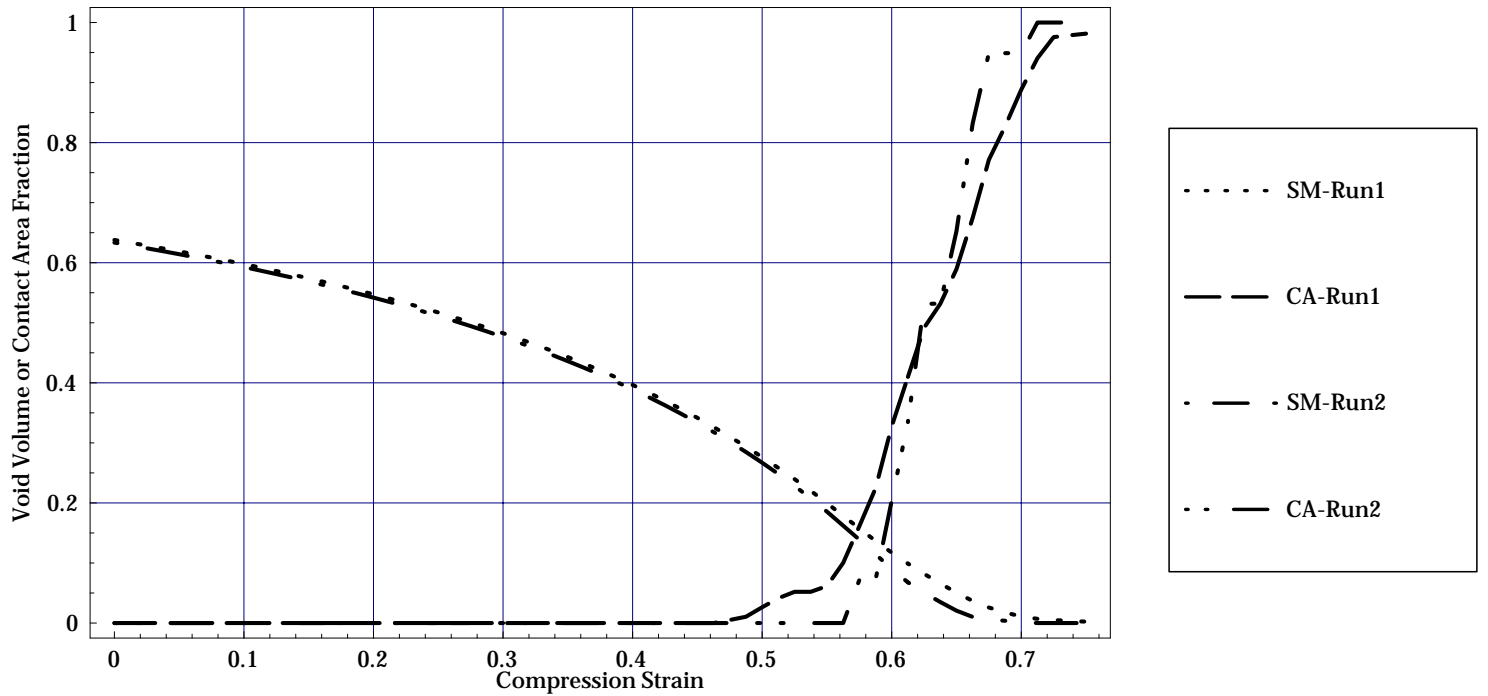

Figure 9.1. The OSB Void Fraction/Contact Area comparison result from two simulation runs. It shows "space/mat" (SM) volume and "contact area" (CA) from those two simulation runs. 


\section{Chapter 10: FUTURE WORK AND CONCLUSIONS}

Of the PSE characteristics mentioned in Chapter 1, experiment management (EM), collaboration support, and high performance computing are the most appealing at the current stage of WBCSim development.

\subsection{Experiment Management}

The development track for the EM component intends to support all simulation models, be more DBMS centric, and be general enough for use in other PSE projects.

(1) Support All Models

Currently, this EM component is only available for two models, RDS and OSB, among the five models described in Chapter 3. RDS was used as the first application of this EM component, since RDS has the smallest number of input and output parameters. Application of the EM component to the OSB model has proved that it can handle the complex OSB model as well. EM support for the other three models (RFP, HC, and CMA) is in progress. Each model will have its own customized comparison functions.

\section{(2) More DBMS Centric Architecture}

Chapter 8 describes the current architecture of WBCSim along with the EM component's role. However, a more database management system-centric architecture (DBMSCA) could provide much more scientific workflow management system functionality. The DBMSCA can define a workflow for the conduct of a WBCSim simulation: preparing the input file, validating the input, invoking the simulation code or submitting a job, formatting files, monitoring the status of a job, and retrieving results. The DBMSCA can then define a schema for the workflow, use a workflow management system that summarizes, keeps track of the status of different jobs, determines what is to be done, triggers upon abnormal conditions (e.g., divide by zero, file not found), etc. A (Postgres) user-defined function looks for records specified in the query. If these records exist, it gets them from the database. If they do not, it "places" some new records into a table called "requests". The workflow manager periodically scans the "requests" table and schedules jobs. When experiments are finished, the manager places appropriate entries in the output tables. This idea has been explored in the $\mathrm{S}^{4} \mathrm{~W}$ PSE for wireless system design [30].

\section{(3) Generalization}

The EM component is specially designed for WBCSim. At VPI, there are currently PSE groups working on cell cycle modeling, wireless communication systems, aircraft design, microarray experiments, and land use change analysis. The usefulness of the EM component for other PSE projects is currently being explored. 


\subsection{Collaboration Support}

Even though the current telnet server and YAPS allow user/guest logins and multiple users at multiple workstations working on WBCSim simultaneously, more sophisticated real-time collaboration support (e.g., Sieve [17]) is needed. This would allow multiple geographically distributed users to jointly modify the same model interface, and concurrently view and annotate the same simulation output. Other features indirectly supporting collaboration are user control over where their data is stored (locally on the client machine vs. remotely on the server), and support for exchanging data with other researchers.

\subsection{High Performance Computing}

Simulation codes used by wood scientists often require access to significant computing resources such as a parallel supercomputer. WBCSim is no exception. The more advanced models in WBCSim, such as the HC Fortran 90 core or its visualization/optimization tools, can take hours to run on a fast (AXP 21064) workstation. By utilizing either parallel or distributed computing, WBCSim can reduce the turnaround time to minutes, making interactive use feasible even for complex three-dimensional models. A planned next step is to use a 200 node Beowulf cluster as a compute engine for WBCSim, transparent to the user, when a requested simulation can be estimated to require such resources.

\subsection{Conclusions}

WBCSim has evolved steadily from a prototype PSE, intended as a tool for computer science PSE research and a Web-based interface for a few legacy computer programs, to a manufacture-oriented near commercial quality PSE that is seriously used by wood science researchers in industry and academia. Since interesting computational capabilities are still lacking (cf. Chapters 10.1-10.3), WBCSim will remain an object of computer science research for some time to come. Yet the program's interfaces, models, and output visualizations are now good enough to be used as production tools by wood scientists. The directions in which computer scientists would like to take WBCSim (collaboration, data mining, grid computing) are quite different from the directions that wood scientists would prefer for WBCSim (new models, refining existing models, more interface and visualization options). The present layered architecture of WBCSim supports these divergent development directions well.

With the addition of the EM component, WBCSim now has a much more efficient tool to manage its simulation execution and experiment data. From the user perspective, the EM component allows a user to easily save, retrieve, compare simulation runs, and further investigate the inter-relationships among multiple parameters. From the developer perspective, the simulation runs are stored in a more systematic manner - a database that permits further annotation and data mining possibilities. Adding a different compare 
function (comparison between simulation outputs) is easy, since only (high level script) wrappers need to be modified.

The original stated goal [14] of WBCSim was to provide "an integrated set of facilities allowing wood scientists to concentrate on high-level problem solving rather than on low-level programming details and application scheduling/execution, allowing users to define, record, and modify problems, and visualize and optimize simulation results". This now seems much closer with the addition of an experiment management capability. 


\section{REFERENCES}

[1] A. Ailamaki, Y.E. Ioannidis, M. Livny, "Scientific workflow management by database management", in Proc. 10th International Conference on Scientific and Statistical Database Management, Capri, Italy, July 1998.

[2] A.L. Ames, D.R. Nadeau, J.L. Moreland, VRML 2.0 Sourcebook, 2nd ed, (John Wiley \& Sons, Inc., New York, 1996).

[3] R.F. Boisvert, J.R. Rice, Solving elliptic problems using ELLPACK, (Springer-Verlag, New York, 1985).

[4] A.J. Bolton, P.E. Humphrey, P.K. Kavvouras, "The hot-pressing of dry-formed woodbased composites. Part III. Predicted vapour pressure and temperature variation with time, compared with experimental data for laboratory boards", Holzforschung, 43 (1989) 265-274.

[5] A.J. Bolton, P.E. Humphrey, P.K. Kavvouras, "The hot-pressing of dry-formed woodbased composites. Part IV. Predicted variation of mattress moisture content with time", Holzforschung, 43 (1989) 345-349.

[6] A.J. Bolton, P.E. Humphrey, P.K. Kavvouras, "The hot-pressing of dry-formed woodbased composites. Part VI. The importance of stresses in the pressed mattress and their relevance to the minimization of pressing time, and the variability of board properties", Holzforschung, 43 (1989) 406-410.

[7] R. Bramley, D. Gannon, T. Stuckey, J. Villacis, E. Akman, J. Balasubramanian, F. Breg, S. Diwan, M. Govindaraju, "The linear system analyzer", Technical Report TR-511, Computer Science Department, Indiana University, Bloomington, IN, 1998.

[8] T. Burnett, C. Chaput, H. Arrighi, J. Norris, D.J. Suson, "Simulating the Glast satellite with Gismo", IEEE Computing in Science and Engineering, 2 (2000) 9-18.

[9] J.X. Chen, X. Fu, "Integrating physics-based computing and visualization: modeling dust behavior", IEEE Computing in Science and Engineering, 1 (1999) 12-16.

[10] C. Dai, P.R. Steiner, "Spatial structure of wood composites in relation to processing and performance characteristics. Part III. Modeling the formation of multi-layered random flake mats", Wood Science and Technology, 28 (1994) 229-239.

[11] E. Gallopoulos, E. Houstis, J.R. Rice, "Computer as thinker/doer: Problem-solving environments for computational science", IEEE Computational Science \& Engineering, 1 (1994) 11-23.

[12] A. Goel, C.A. Baker, C.A. Shaffer, B. Grossman, W.H. Mason, L.T. Watson, R.T. Haftka, "VizCraft: a problem-solving environment for aircraft configuration design", IEEE Computing in Science and Engineering, 3 (2001) 56-66.

[13] A. Goel, C. Phanouriou, F.A. Kamke, C.J. Ribbens, C.A. Shaffer, L.T. Watson, "WBCSim: a prototype problem solving environment for wood-based composites simulations", Technical Report TR98-25, Computer Science Department, Virginia Polytechnic Institute and State University, Blacksburg, VA, 1998.

[14] A. Goel, C. Phanouriou, F.A. Kamke, C.J. Ribbens, C.A. Shaffer, L.T. Watson, "WBCSim: a prototype problem solving environment for wood-based composites simulations", Engineering with Computers, 15 (1999) 198-210.

[15] C.R. Haselein, "Numerical simulation of pressing wood-fiber composites", Ph.D. Dissertation, Forest Products Dept., Oregon State University, Corvallis, OR, 1998. 
[16] E.N. Houstis, A.C. Catlin, J.R. Rice, V.S. Verykios, N. Ramakrishnan, C.E. Houstis, "PYTHIA-II: a knowledge/database system for managing performance data and recommending scientific software", ACM Transactions on Mathematical Software, 26 (2000) $227-253$.

[17] P.L. Isenhour, J. Begole, W.S. Heagy, C.A. Shaffer, "Sieve: A Java-based collaborative visualization environment", in Late Breaking Hot Topics Proceedings, IEEE Visualization '97, (Phoenix, AZ, 1997) 13-16.

[18] Y.E. Ioannidis, M. Livny, S. Gupta, N. Ponnekanti, "Zoo: a desktop experiment management environment", in Proc. 22nd International VLDB Conference, Bombay, India, September 1996, 274-285.

[19] F.A. Kamke, J.B. Wilson, "Computer simulation of a rotary dryer: retention time", American Institute of Chemical Engineers J., 32 (1985) 263-268.

[20] F.A. Kamke, J.B. Wilson, "Computer simulation of a rotary dryer: heat and mass transfer", American Institute of Chemical Engineers J., 32 (1985) 269-275.

[21] M.E. Lang, M.P. Wolcott, "Modeling the consolidation of wood-strand mat", Mechanics of Cellulosic Materials, ASME AMD 209/MD 60 (1995) 153-176.

[22] M.E. Lang, M.P. Wolcott, "A model for viscoelastic consolidation of wood-strand mats. Part I. Structural characterization of the mat via Monte Carlo Simulation", Wood and Fiber Science, 28 (1996) 100-109.

[23] M.E. Lang, M.P. Wolcott, "A model for viscoelastic consolidation of wood-strand mats. Part II. Static stress-strain behavior of the mat", Wood and Fiber Science, 28 (1996) 369-379.

[24] C. Lu, "Organization of wood elements in partially oriented flakeboard mats", Ph.D. Dissertation. Dept. of Forestry, University of British Columbia, Vancouver, BC, 1999.

[25] S.G. Parker, D.M. Weinstein, C.R. Johnson, "The SCIRun computational steering software system", in Modern Software Tools in Scientific Computing, E. Arge, A. M. Bruaset, H. P. Langtangen (eds.), (Birkhauser Press, 1997) 1-40.

[26] J. Resnik, F.A. Kamke, "Modeling the cure of adhesive-wood bonds using high frequency energy", Final Report, U.S.-Slovene Joint Board on Scientific and Technological Cooperation, Project 95-AES10, University of Ljubljana, Ljubljana, Slovenia, 1998.

[27] C.A. Shaffer, L.T. Watson, D.G. Kafura, N. Ramakrishnan, "Features of problem solving environments for computational science", in Proc. High Performance Computing Symp. 2000, A. Tentner (ed.), (Soc. for Computer Simulation Interna, San Diego, CA, 2000) $242-247$.

[28] H. Thöemen, "Modeling the physical processes in natural fiber composites during batch and continuous pressing", Ph.D. dissertation. Forest Products Dept., Oregon State University, Corvallis, OR, 2000.

[29] Vanderplaats Research \& Development, Inc., DOT Users Manual, Version 4.20, (Colorado Springs, CO, 1985).

[30] A. Verstak, "Data and Computation Modeling for Scientific PSEs", M.S. Thesis, Virginia Polytechnic Institute and State University (CS), Aug 2002.

[31] A. Verstak, M. Vass, N. Ramakrishnan, C. Shaffer, L. Watson, K.K. Bae, J. Jiang, W. H. Tranter, T.S. Rappaport, "Lightweight data management for compositional modeling in problem solving environments", Proc. High Performance Computing Symp. 2001, A. Tentner (ed.), (Soc. for Computer Simulation Int., San Diego, CA, 2001) 148-153. 
[32] S. Wolfram, The Mathematica Book, 3rd ed., (Wolfram Media/Cambridge University Press, 1996).

[33] B.G. Zombori, "Modeling the transient effects during the hot-pressing of wood-based composites", Ph.D. Dissertation, Virginia Polytechnic Institute and State University, Blacksburg, VA, 2001.

[34] B.G. Zombori, F.A. Kamke, L.T. Watson, "Simulation of the mat formation process", Wood Fiber Science, 33 (2001) 564-580. 


\section{Appendix A: Perl Subroutine gencontourplot}

The following subroutine gencontourplot is one of the subroutines in the Perl script OSBsub.pl called by the OSB Wrapper OSB.pl. This subroutine illustrates that the current WBCSim approach keeps the Mathematica code as a blueprint external to the wrapper. Then in every simulation run, the wrapper makes a copy of that blueprint code, uses sed to insert the dynamic graphics output directory, and then feeds it directly to the Mathematica kernel, which is discussed in Chapter 6.

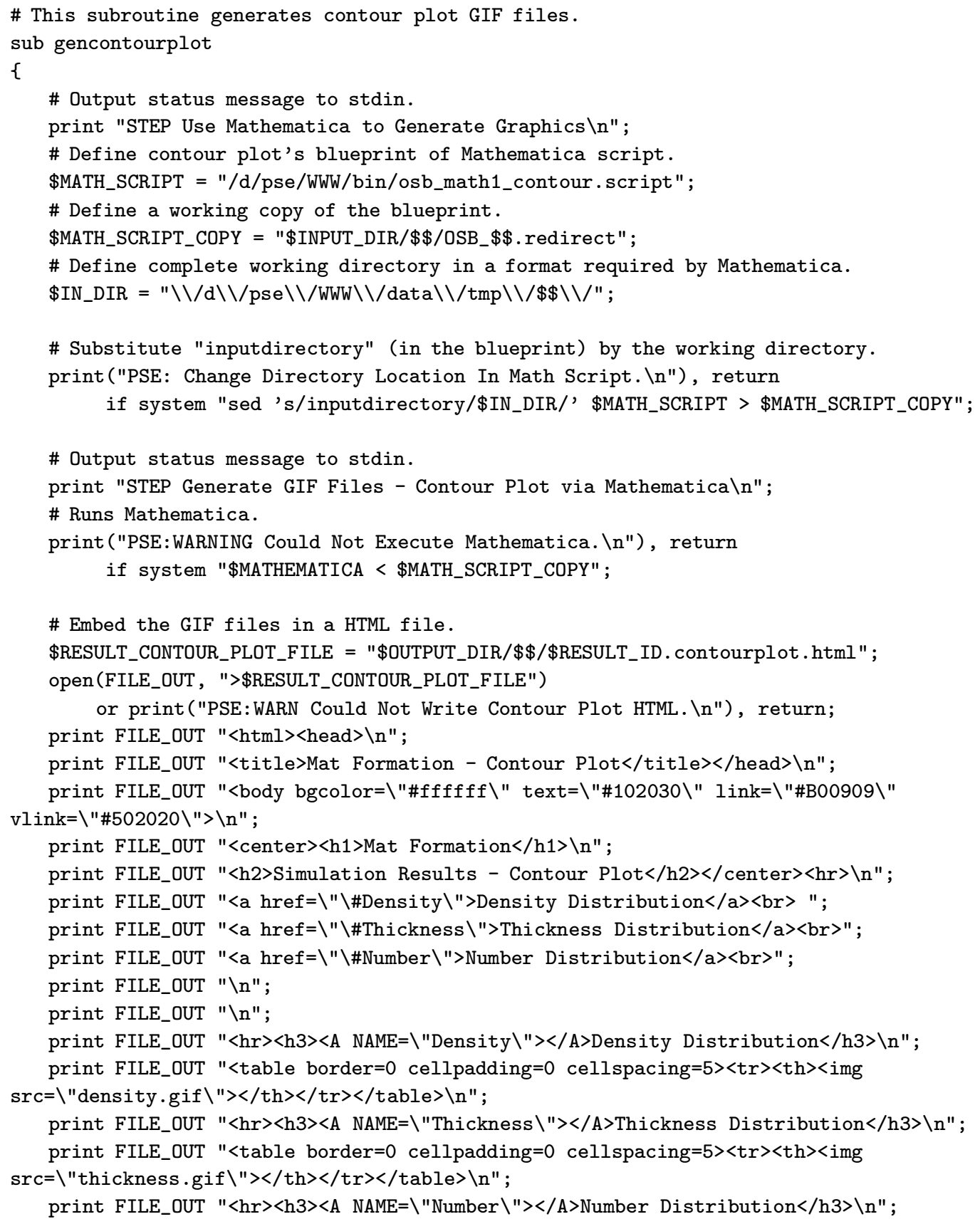


print FILE_OUT " $\langle$ table border=0 cellpadding=0 cellspacing=5 $\rangle\langle$ tr $\rangle\langle$ th $\rangle\langle$ img src $=\backslash$ "number.gif $\backslash "\rangle\langle/$ th $\rangle\langle/$ tr $\rangle\langle/$ table $\rangle \backslash \mathrm{n} "$;

print FILE_OUT " $\langle\mathrm{hr}></$ body $></ \mathrm{html}>\backslash \mathrm{n} "$;

close(FILE_OUT)

or print("PSE:WARN Could Not Write Graph HTML. \n"), return;

\# Output result HTML filename and related info.

\&PrintURL("\$RESULT_CONTOUR_PLOT_FILE", "text/html", "Contour Plot");

\} 


\section{Appendix B: Perl Code Example of WhirlGif from gen3Dplot}

The following segment of code is from the subroutine gen3Dplot, which is one of the subroutines in the Perl script HOTsub.pl called by the HOT wrapper HOT.pl.

The code illustrates how WBCSim uses the the UNIX utility WhirlGif to generate a single animated GIF image file from a sequence of GIF image files, as described in Chapter 6 .

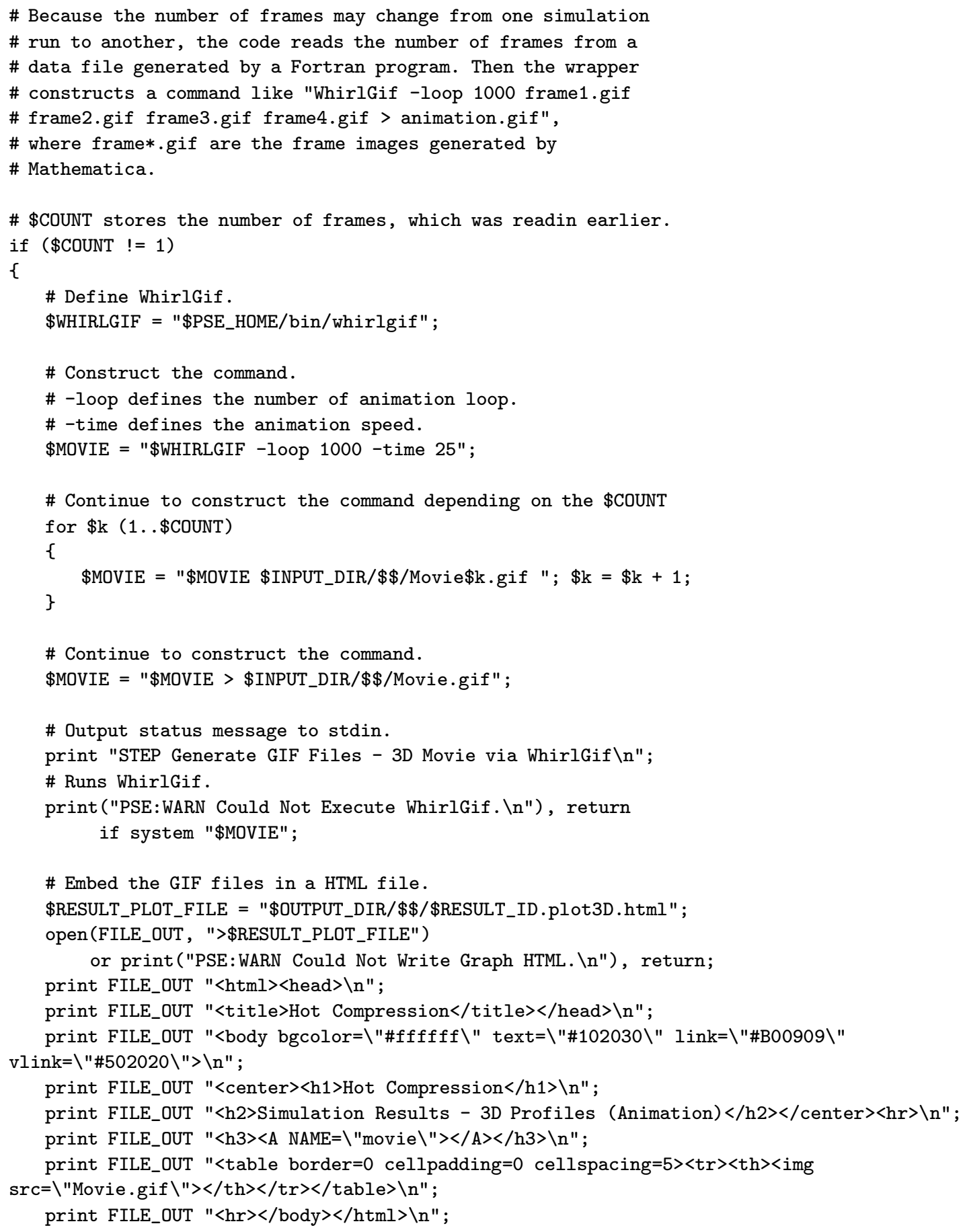


close(FILE_OUT)

or print("PSE:WARN Could Not Write Graph HTML. \n"), return;

\# Output result HTML filename and related info.

\&PrintURL("\$RESULT_PLOT_FILE", "text/html", "3D Profiles (Animation)");

\} \# End of if 


\section{Appendix C: Perl OSB Save Wrapper osbsave.pl}

The following Perl script is the OSB save wrapper osbsave.pl, which illustrates how WBCSim interacts with the database to save inputs and outputs, as discussed in Chapter 8 .

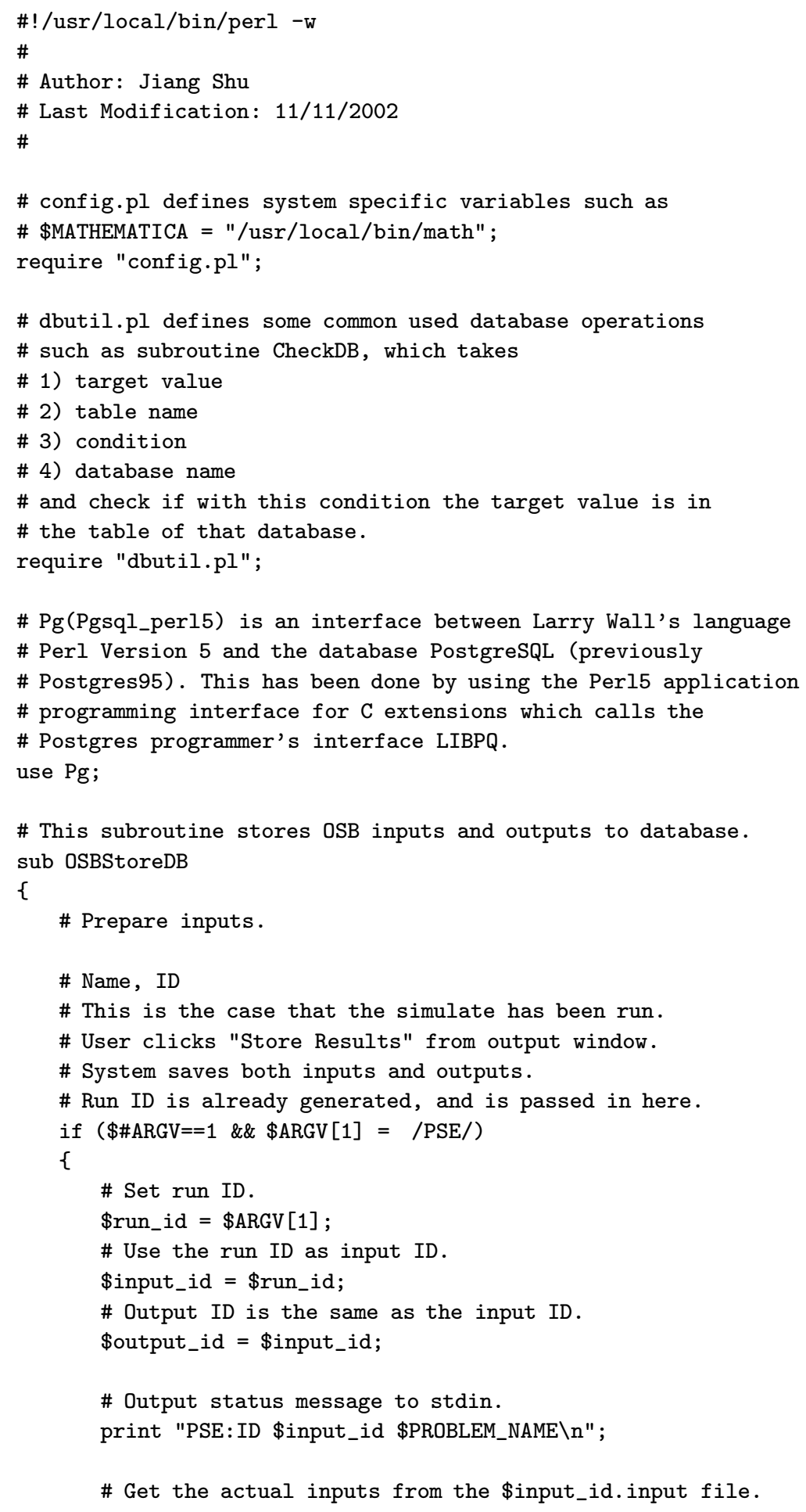




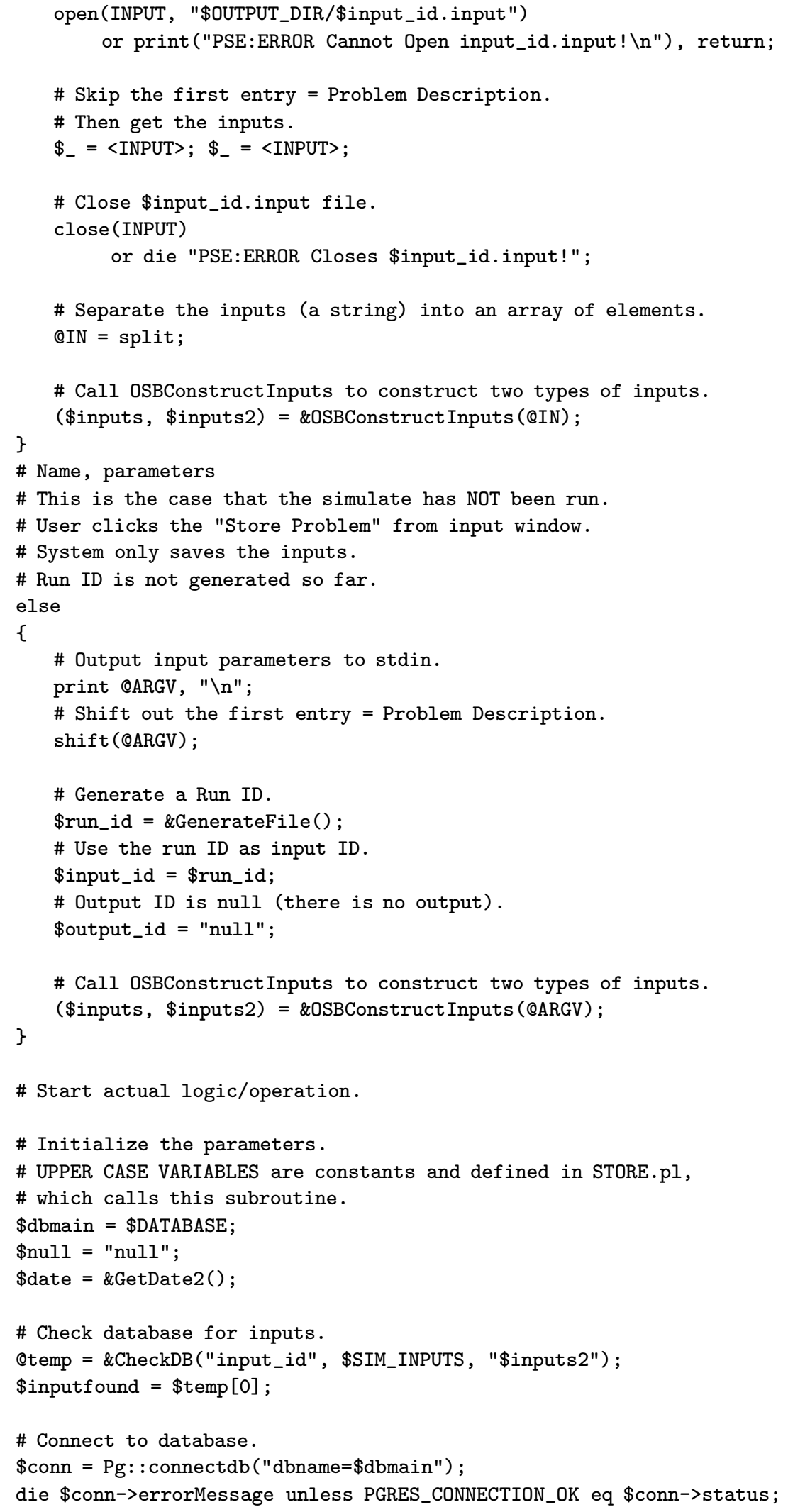




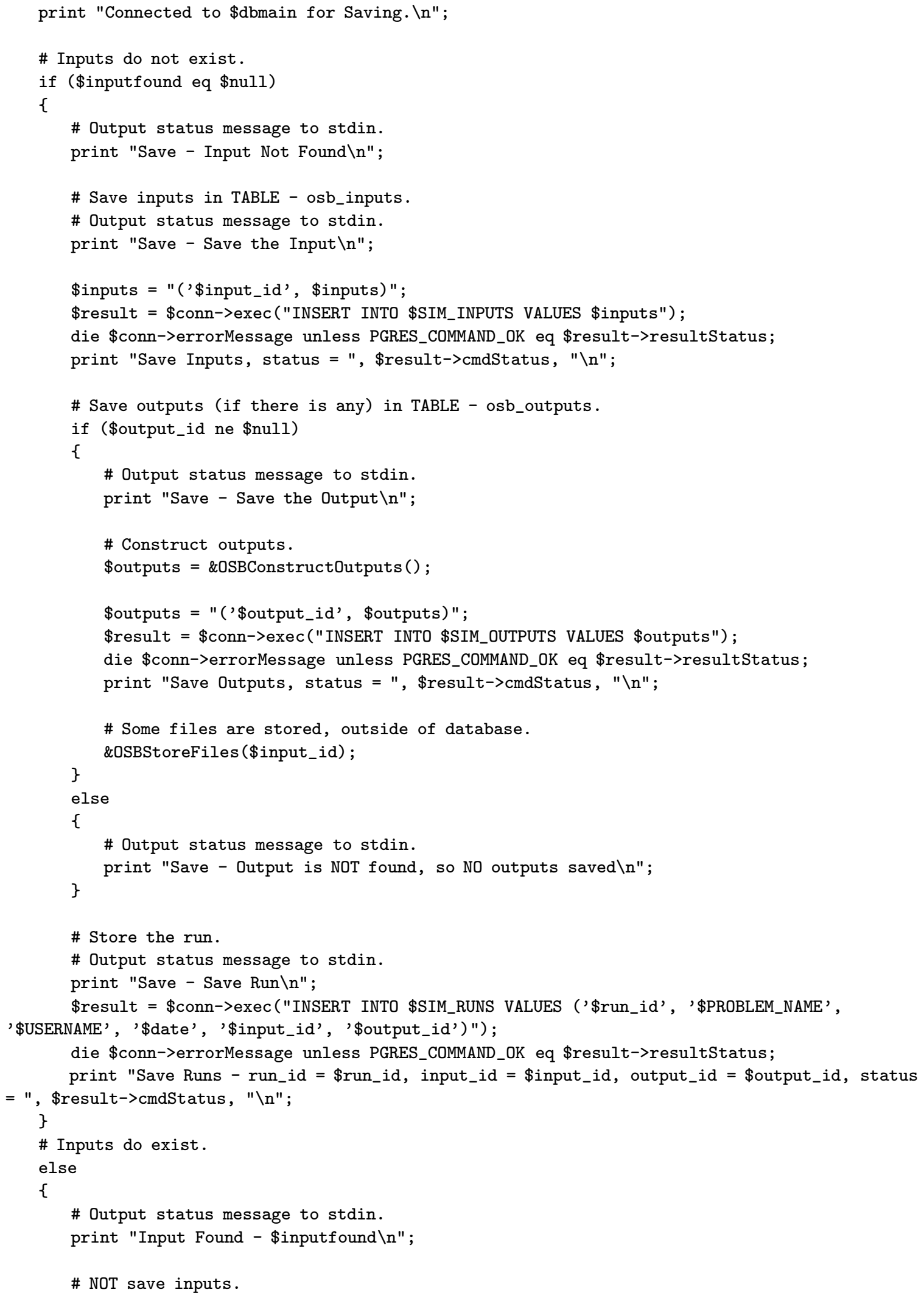




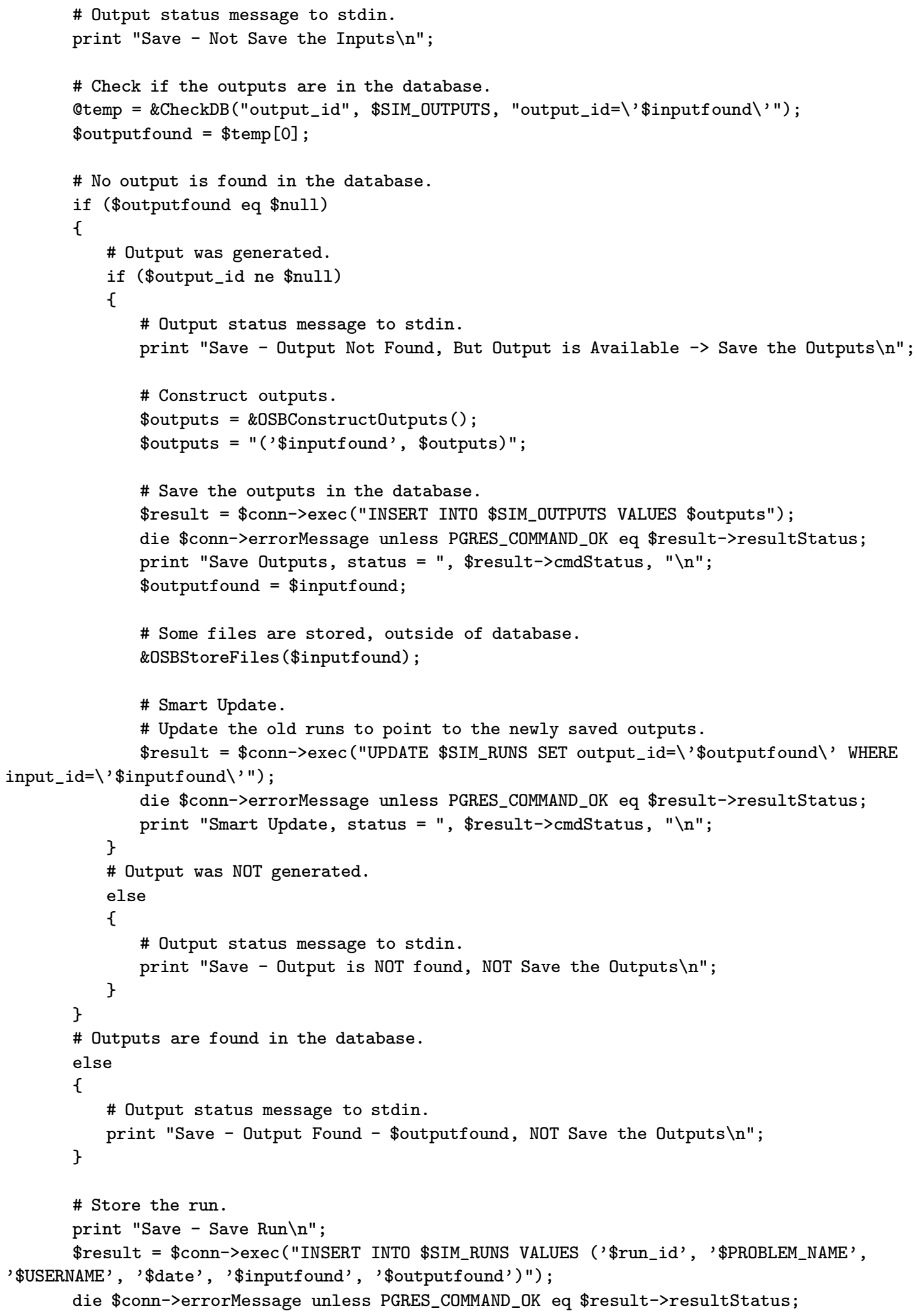


print "Save Runs - run_id = \$run_id, input_id = \$inputfound, output_id = \$outputfound, status = ", \$result->cmdStatus, "\n";

\}

\} \# End of OSBStoreDBsub

\# This subroutine constructs two types of inputs.

\#1) \$inputs = just the parameters

\# 2) $\$$ inputs2 = inputs with column names for the query

sub OSBConstructInputs

\{

\# Initialize the parameters.

$\$ i=0$;

\$inputs $="$ ";

\$inputs2 = " ";

\# First few column names in the table.

@p = ("dimension", "layers", "seed", "mat_d1", "mat_d2", "nmp1", "nmp2", "cut1", "cut2", "noi", "pn");

\# Get @p data.

for $\$ i(0 . .10)$

\{

$\$ a=\operatorname{shift}\left(\varrho_{-}\right) ;$

\$inputs = "\$inputs\$a, ";

\$inputs2 = "\$inputs $2 \$ \mathrm{p}[\$ \mathrm{i}]=\$ \mathrm{a}$ and $"$;

\# Get dimension (2D or 3D).

if $(\$ i==0)$

\{

\}

\$dimension $=\$ a ;$

\# Get number of layers.

if $(\$ i==1)$

\{

\}

\$number_of_layers $=\$ a$;

$\$ i++$;

\}

\# Get number of press.

$\$ \mathrm{pn}=2 * \$ \mathrm{a}$;

\$pndata $="\{"$;

$\$ i=1$;

for $\$ \mathrm{i}(1 \ldots \mathrm{pn})$

\{

$\$ a=\operatorname{shift}\left(\varrho_{-}\right) ;$

\$pndata $=$ "\$pndata $\backslash "$ a $\backslash ", "$;

\$i++;

\}

chop (\$pndata); chop (\$pndata);

\$pndata $=$ "\$pndata $\}$;

\$inputs = "\$inputs \'\$pndata \', ";

$\$ \mathrm{x}=$ "pressdata";

\$inputs2 = "\$inputs2\$x=\'\$pndata $\backslash$ ' and "; 


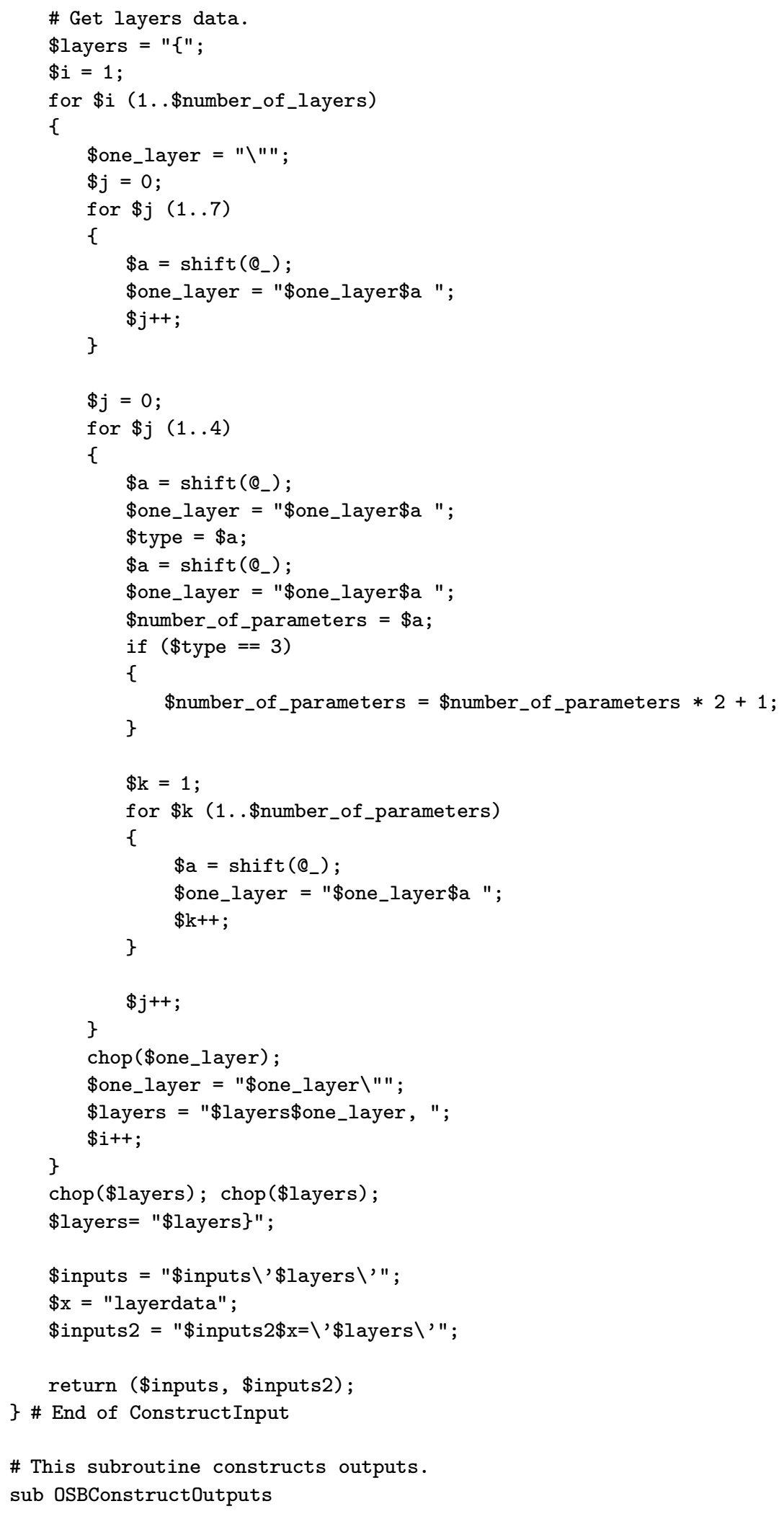




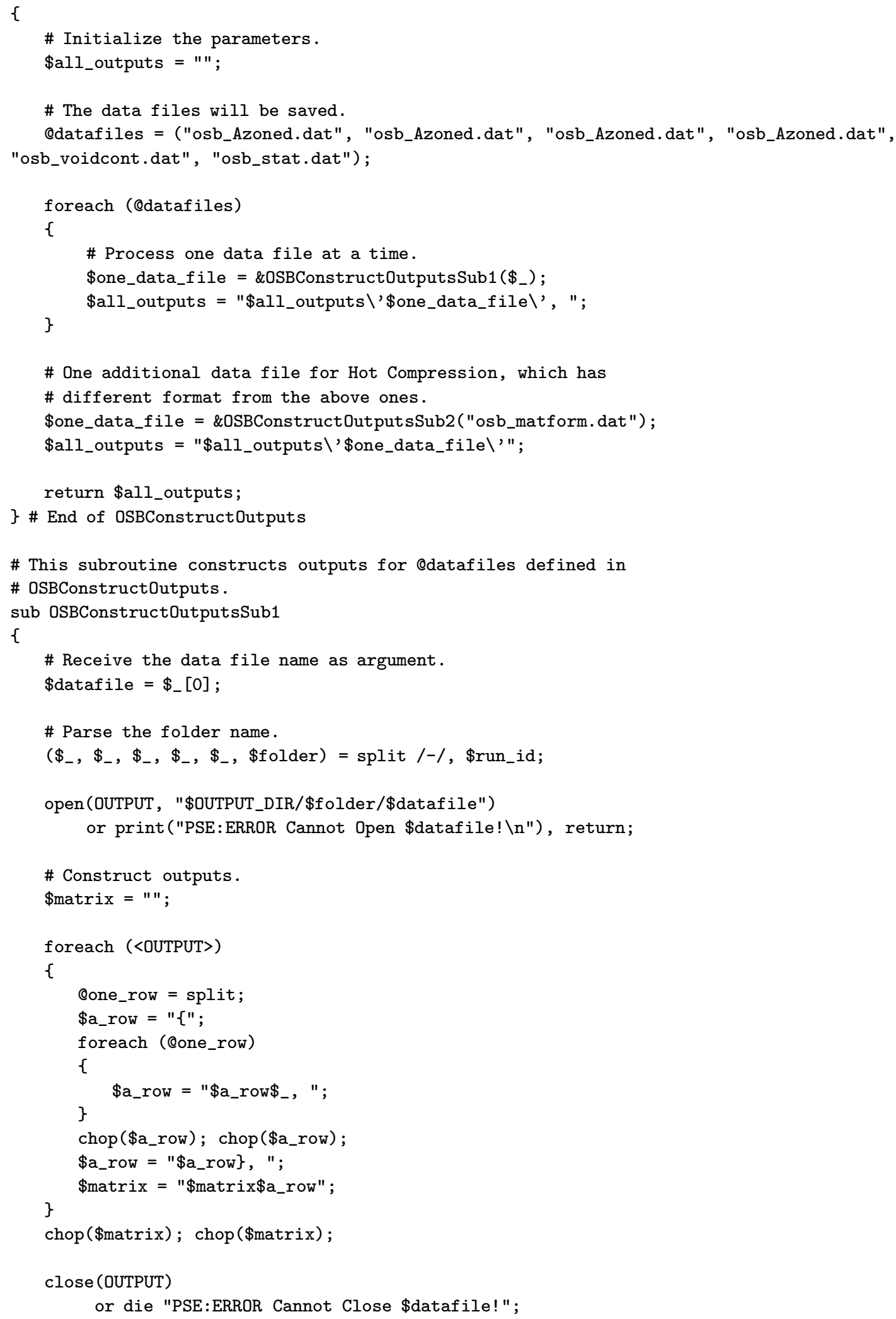


return " $\{\$$ matrix $\} " ;$
\# End of OSBConstructOutputsSub1

\# This subroutine constructs outputs for osb_matform.dat, \# which has different format from the other files. sub OSBConstructOutputsSub2 \{

\# Receive the data file name as argument.

\$datafile $=\$$ _ $[0]$;

\# Parse the folder name.

$\left(\$_{-}, \$_{-}, \$_{-}, \$_{-}, \$_{-}, \$\right.$ folder $)=$ split $/-/$, \$run_id;

open(OUTPUT, "\$OUTPUT_DIR/\$folder/\$datafile")

or print("PSE:ERROR Cannot Open \$datafile! \n"), return;

\# Construct outputs.

\$one_line = " ";

$<$ OUTPUT> ;

foreach (<OUTPUT $>)$

\{

Qone_row = split;

if (\$one_row[0] ne "/")

\{

\$one_line = "\$one_line\$one_row [1], ";

\}

\}

chop(\$one_line); chop(\$one_line);

close(OUTPUT)

or die "PSE:ERROR Cannot Close \$datafile!";

return "\{\$one_line $\} "$;

\} \# End of OSBConstructOutputsSub2

\# Some files are stored outside of the database such as:

\# 1) The descriptive input file, which only helps a user to understand the input file.

\# 2) 2D and 3D layer images, which are only for visual effect.

\# datafiles (containing coordinates of flakes)

\# are not saved in the database.

sub OSBStoreFiles

\{

\# Receive ID as argument.

$\$ i d=\$[0]$;

\# Parse the folder name.

$\left(\$_{-}, \$_{-}, \$_{-}, \$_{-}, \$_{-}, \$\right.$ folder $)=\operatorname{split~} /-/$, \$output_id;

\# Save the descriptive input file.

print("PSE:ERROR Copying 2D pictures. $\backslash n ")$, exit 2

if system "\$CP \$OUTPUT_DIR/\$folder/osb_input.dat \$SIMDIR/\$id.osb_input.dat";

\# Save 2D Gif Files. 


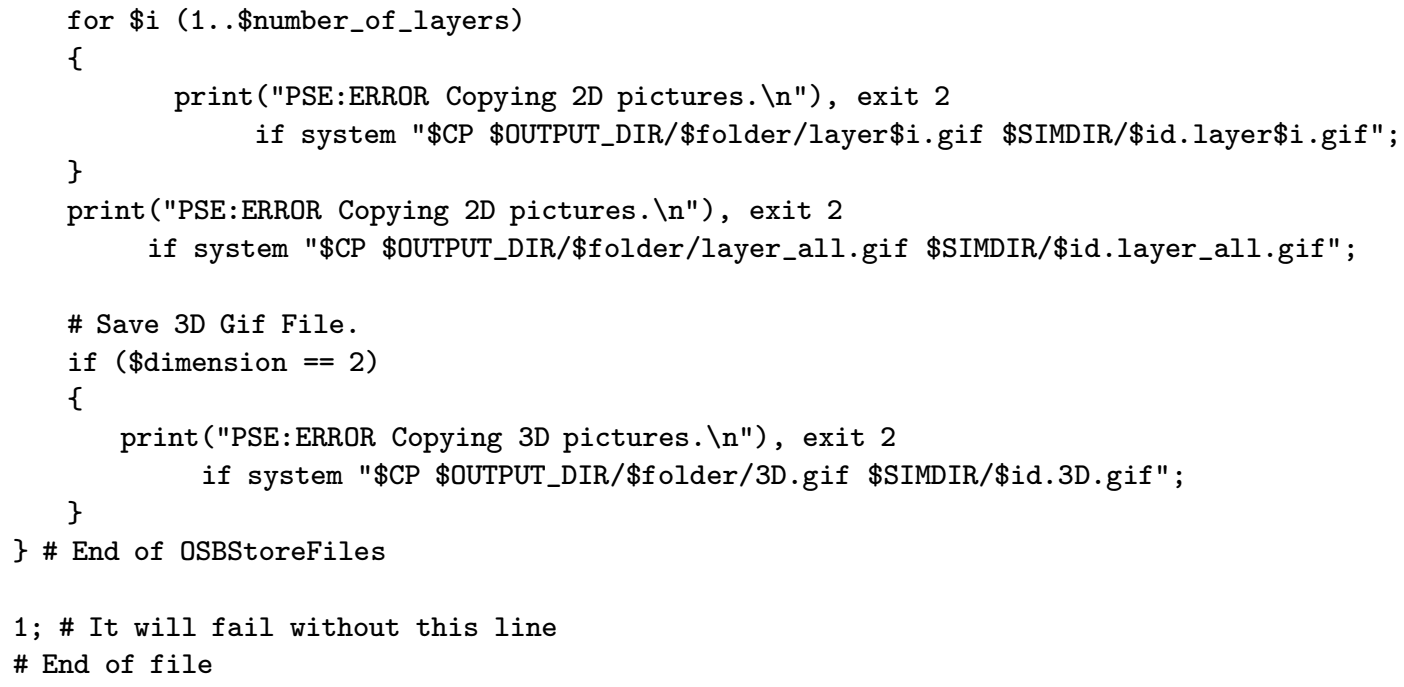




\section{Appendix D: Perl OSB Load Wrapper osbload.pl}

The following Perl script is the OSB load wrapper osbload.pl, which illustrates how WBCSim interacts with the database to load saved simulation runs, as discussed in Chapter 8 .

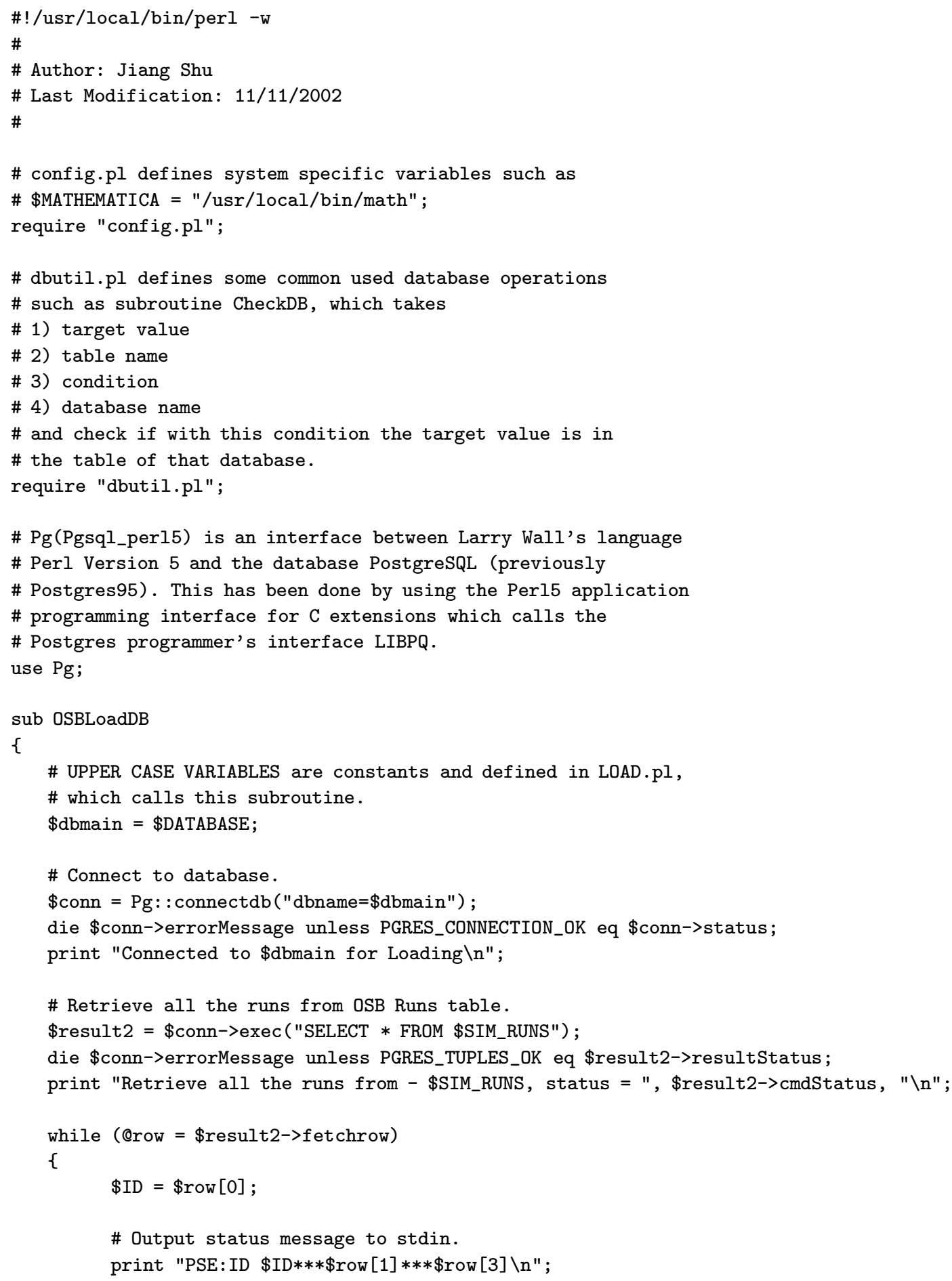


\# Print archive URL.

print "PSE:URL http://wbc.forprod.vt.edu/ pse/data/tmp/\$ID. input $x-w b c / x-a r c h i v e$ Archive File $\backslash n "$;

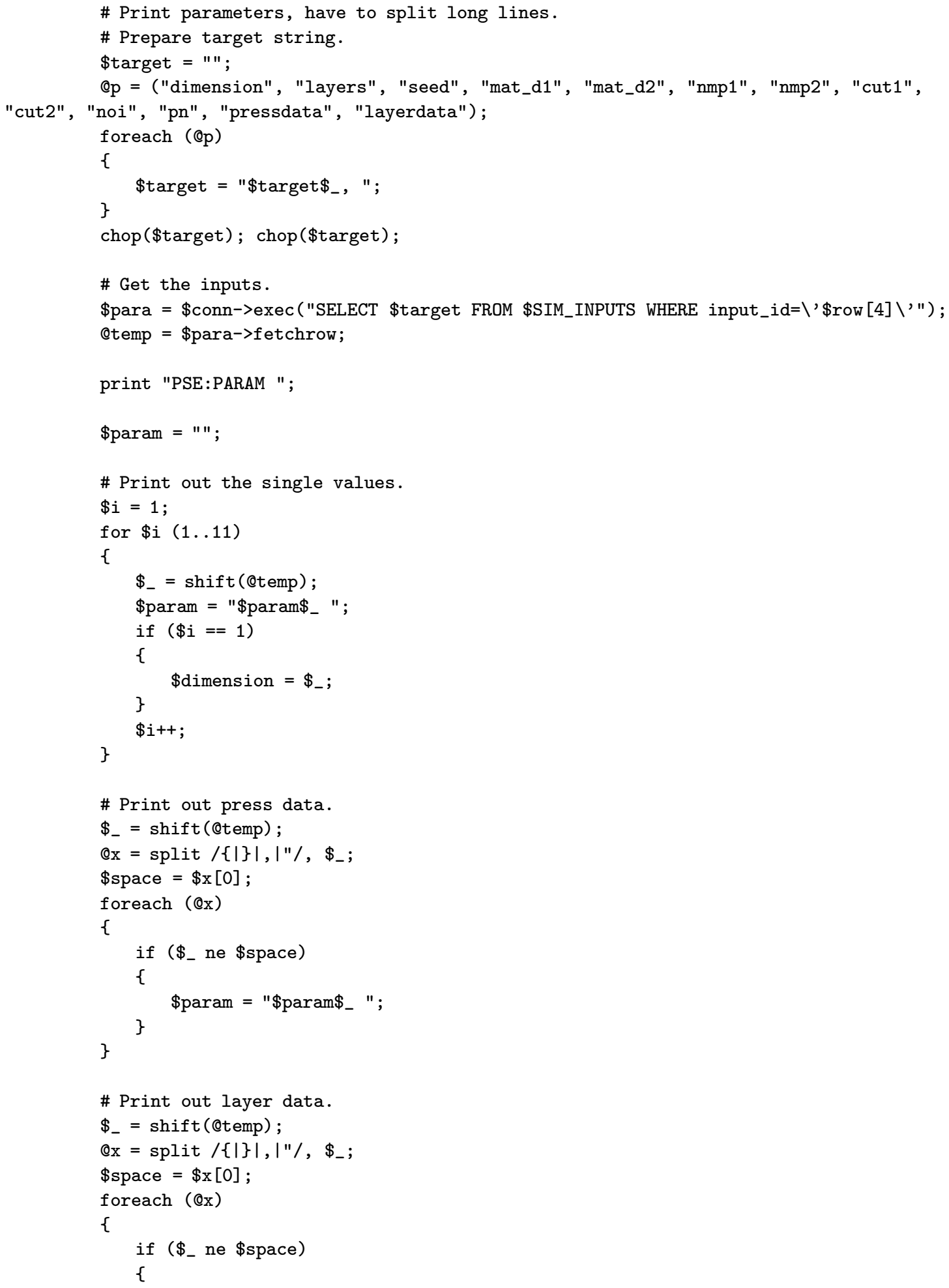




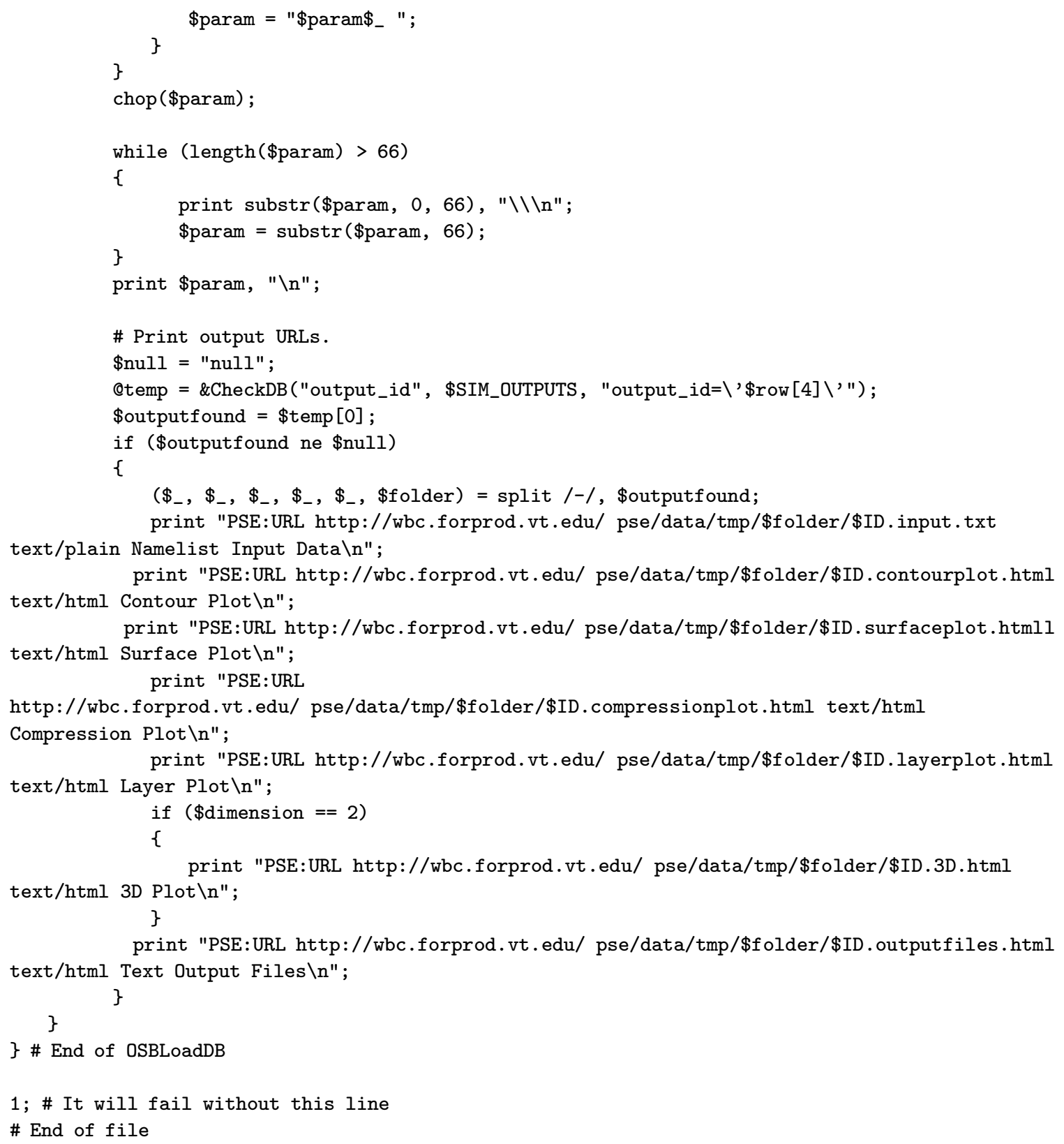




\section{VITA}

Jiang Shu was born in Wuhan, P. R. China, on July 3rd, 1976. He earned two bachelor's degrees, in Computer Science and Mathematics, in May 2000 from University of Louisiana at Monroe, Monroe, Louisiana, USA. In August 2000, he started graduate study in computer science at Virginia Polytechnic Institute and State University. In October 2002, he received the Master of Science degree in computer science from Virginia Polytechnic Institute and

State University. He will continue his study in pursuit of the Doctor of Philosophy degree in computer science. 